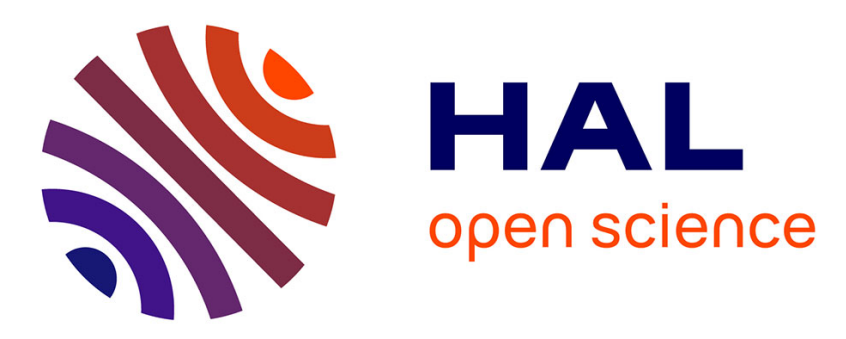

\title{
Market Value Margin calculations under the Cost of Capital approach within a Bayesian chain ladder framework
}

Christian Robert

\section{- To cite this version:}

Christian Robert. Market Value Margin calculations under the Cost of Capital approach within a Bayesian chain ladder framework. Insurance: Mathematics and Economics, 2013, 10.1016/j.insmatheco.2013.05.003 . hal-00618013

\section{HAL Id: hal-00618013 https://hal.science/hal-00618013}

Submitted on 31 Aug 2011

HAL is a multi-disciplinary open access archive for the deposit and dissemination of scientific research documents, whether they are published or not. The documents may come from teaching and research institutions in France or abroad, or from public or private research centers.
L'archive ouverte pluridisciplinaire HAL, est destinée au dépôt et à la diffusion de documents scientifiques de niveau recherche, publiés ou non, émanant des établissements d'enseignement et de recherche français ou étrangers, des laboratoires publics ou privés. 


\title{
Market Value Margin calculations under the Cost of Capital approach within a Bayesian chain ladder framework
}

\author{
Christian Y. ROBERT* \\ Université de Lyon, Université Lyon 1, Institut de Science Financière et d'Assurances, \\ 50 Avenue Tony Garnier, F-69007 Lyon, France
}

\begin{abstract}
In the Solvency II framework, insurance companies need to calculate the Best Estimate valuation of Liabilities (BEL) and the Market Value Margin (MVM) for non-hedgeable insurancetechnical risks. The Cost-of-Capital approach defines the MVM as the present value of the current and future Solvency Capital Requirement (SCR) of the non-hedgeable risks to protect against adverse developments in the run-off of the insurance liabilities. However the SCR at time $t$ itself depends on the increase in the MVM between $t$ and $t+1$. Hence there exists an intricate circularity dependency between both quantities. In this paper we present exact and accurate approximate analytic formulas for MVMs within a Bayesian log-normal chain ladder framework.
\end{abstract}

\section{Introduction}

Solvency II is creating a new approach to regulate capital requirements by quantifying risks and is giving incentives for companies to develop good risk management practices. It will be based on economic principles for the measurement of assets and liabilities and capital requirements will depend directly on this. In particular it introduces the market consistent economic (solvency) balance sheet and two points in time are considered to calculate the Solvency II Capital Requirement (SCR): the current balance sheet and the balance sheet at the end of the year. The main components of this balance sheet are the Market Value of Assets (MVA) and the Fair Value of Liabilities (FVL) consisting of the sum of two components: the Best Estimate valuation of Liabilities (BEL) and the Market Value Margin (MVM).

The BEL is the present value of expected future cash flows using best estimate assumptions with no explicit margins incorporated. However, for the non-hedgeable insurance risks, since there exists the risk that actual experience will be more adverse than expected, there needs to be an additional "risk margin" component added to the BEL, the MVM. It can be interpreted as the cost of risk and uncertainty in the amount and timing of future payments needed to satisfy insurance liabilities.

For several decades, actuaries have used a variety of technical methods to consider the risk in the valuation of their insurance liabilities and risk margins have been implicitly or explicitly embedded in the assumptions or in the methods. Approaches for determining risk margins have been grouped into four families (see IAA position paper [9])

${ }^{*}$ This work has been partially supported by the BNP Paribas Insurance Chair «Management de la modélisation». The views expressed in this document are the authors own and do not necessarily reflect those endorsed by BNP Paribas Insurance. 
- the quantile methods that use risk measures such as the Value-at-Risk (VaR), the Conditional Tail Expectation (CTE) and the Tail Value-at-Risk (TVaR) and define the risk margin as the difference of the risk measure of the discounted ultimate future payments and the BEL;

- the Cost-of-Capital ( $\mathrm{CoC})$ approach that defines the risk margin as the present value of the current and future SCRs for the non-hedgeable risks to protect against adverse developments in the run-off of the insurance liabilities;

- the discount related methods that define the risk margin as the difference of the discount expected cash flows using the risk-free interest rate minus a selected risk adjustment and the BEL (probability distortions to take into account risk aversion are alternatively used in [18]);

- the methods that use explicit assumptions: the risk margin results from selecting prudent explicit parameters and simpler methodologies.

Over the recent years, the $\mathrm{CoC}$ approach has been preferred to estimate market-consistent risk margins for insurance contracts. One of the reason is that $\mathrm{CoC}$ approach is commonly used as a conceptual framework in both non-life and life insurance valuation applications. Solvency II prescribes that the MVM is calculated using the following 3 steps:

1. determine the expected SCRs for non-hedgeable risks until the run-off of the portfolio (remind that the SCR is defined as the amount of capital required to support the claims paid out and the increase in the sum of the BEL and MVM following a one-in-200-year event over the next year);

2. calculate the capital charge as the SCRs multiplied by the CoC charge and take the present value of the product;

3. take the sum of the present values for all years until the run-off to arrive at the MVM.

As can be read in this approach, estimating the SCRs for each future year in a theoretically correct way is far from straightforward because the SCRs appear to depend on the MVMs, and the MVMs depend on the SCRs. Hence there exists an intricate circularity dependency between both quantities. To mitigate the circularity issue, several simplifications have been suggested (from the more complex to the simpler):

- exclude the risk margin in the FVL within the calculations for the SCRs (see e.g. the Swiss Solvency Test);

- approximate the SCRs for the future years by using a 'proportional proxy': for example the current SCR is calculated, but the future SCRs are approximated by multiplying the current SCR by the ratio of the future BEL to the current BEL;

- approximate the current MVM by using a 'duration approach': the MVM is calculated as the current SCR multiplied by a modified duration of the insurance liabilities (see e.g. [13]);

- approximate the current MVM by considering it as a percentage of the current BEL.

The problem of calculating the MVM in a theoretically correct way can only be solved by starting at the final time period and working backwards recursively. The problem is in general intractable without simplifications. 
In [7], Haslip considers the liabilities of a non-life insurance company and assumes that the outstanding claims reserve at the end of each year is subject to uncertainty through a scaling factor drawn from a log-normal distribution with mean 1 and several sets of coefficients of variation. He makes some comparisons with proxies derived from the proportional method, the duration method or by considering the MVM as a percentage of the current BEL. The results are: the duration method understates the risk margin, the proportional method provides a reasonable approximation but sensitive to the coefficients of variation of the log-normal distributions, the 'percentage of BEL' proxy is more conservative than the others.

In [1] and [4], Bonnard, Daya and Margetts alternately assume that the cumulative paid claims (for all the accident years) at the end of each year are equal to the cumulative paid claims at the beginning of the year multiplied by a scaling factor drawn from a log-normal distribution with deterministic but path dependent mean and volatility. They find that the first approximation (i.e. excluding the risk margin in the FVL within the calculations for the SCRs) gives higher results than the exact solution. The second and the third approximations (proportional and duration proxies) tend to underestimate the exact solution. The fourth approximation can be quite larger than the analytical solutions specially for the first development years.

In [14], Salzmann and Wüthrich derive analytical formulas for the risk margin and compare different proxies but under the assumption that the capital requirements are defined through the standard error of the sum of the claims paid out and the increase in the BEL and the risk margin instead of the 99.5th percentile of the change as needed for the Solvency II approach.

In this paper, we consider a Bayesian log-normal chain ladder model for a non-life insurance company and extend the results of Bonnard, Daya and Margetts to the case (i) where recent information is immediately absorbed by the Bayesian model, (ii) where the cumulative paid claims of each accident year follow a multiplicative log-normal model and/or (iii) where the cash flows and the SCRs are discounted within the calculation of the BEL and the MVM. We give an exact analytical formula for the MVM of the liabilities of a specific accident year when reserves are not discounted. We propose accurate approximated analytic formulas for the MVM of the liabilities of all accident years (when reserves are discounted or not) by considering convex order techniques and approximations as used in [5], [6], [15], [16].

The paper is organized as follows. In Section 2 we define the Bayesian log-normal chain ladder model for claims reserving and calculate the BEL depending on whether or not the cash flows are discounted. We then give general results concerning convex order approximations of sums of log-normal random variables in Section 3. In Section 4 and 5 we provide respectively recursive analytical formulas for the calculation of the MVM when cash flows are not discounted or when they are subject to a constant discount rate. Finally, in Section 6 we provide a real data example that is based on liability insurance data. The proofs are deferred to Section 7 .

\section{Bayesian log-normal chain ladder model}

We consider the liabilities of a non-life insurance company. The random variables $C_{i, j}>0$ denote the cumulative payments of accident year $i \in\{1, \ldots, I\}$ after development year $j \in\{0, \ldots, J\}$, meaning that the incremental claims, $X_{i, j}=C_{i, j}-C_{i, j-1}$ for $j=1, \ldots, J$, are paid in calendar (accounting) year $k=i+j$. We assume that all claims are settled after development $J$ and that $I \geq J+1$. We define the individual claims factor $F_{i, j}=C_{i, j} / C_{i, j-1}$ for $j=1, \ldots, J$ and let $\xi_{i, j}=\log \left(F_{i, j}\right)$. The first payment $C_{i, 0}$ is the initial value of the process $\left(C_{i, j}\right)_{j=0, \ldots, J}$ and $F_{i, j}$ are the multiplicative changes. 
The time unit corresponds to years, the current year is $I$ and we write

$$
\mathcal{T}_{t}=\sigma\left\{X_{i, j}: i+j \leq t, i=1, \ldots, I, j=0, \ldots, J\right\}
$$

for $t=I, \ldots, I+J$.

We consider a Bayesian log-normal chain ladder model to complete the run-off trapezoid. For the claims reserving problem, Bayesian methods are now well investigated (see e.g. [17], [2]) and they provide an interesting approach for a successive information update in each accounting year. Log-normal chain ladder models have been introduced by Hertig [8] and their Bayesian versions have been recently used by Merz and Wüthrich [12] to model paid-incurred chain claims. We restrict ourselves to this model because it allows for explicit analytical formulas for the MVM of the liabilities of a specific accident year when reserves are not discounted.

We assume that

- given $\boldsymbol{\Phi}=\left(\Phi_{0}, \ldots, \Phi_{J-1}\right)$ and $\boldsymbol{\sigma}=\left(\sigma_{0}, \ldots, \sigma_{J-1}\right)$, the $X_{i, j}$ are independent for different accident years $i$, and for $j=0, \ldots, J-1$, the $\xi_{i, j+1}$ are independent and satisfy $\xi_{i, j+1} \sim$ $\mathcal{N}\left(\Phi_{j}, \sigma_{j}^{2}\right)$

- $\boldsymbol{\sigma}>0$ is deterministic and the $\Phi_{j}$, for $j=0, \ldots, J-1$, are independent normally distributed, $\Phi_{j} \sim \mathcal{N}\left(\phi_{j}, s_{j}^{2}\right)$, with prior parameters $\phi_{j}$ and $s_{j}^{2}>0$;

- $\left(C_{1,0}, \ldots, C_{I, 0}\right)$ and $\boldsymbol{\Phi}$ are independent.

This model is close to the normal-normal Bayes chain ladder model and belongs to the exponential dispersion family with associate conjugate priors (see e.g. [3]). It leads to exact formulas for the calculation of the BEL but the Bayesian estimators do not coincide with the linear credibility estimators.

At time $t$ we have information $\mathcal{T}_{t}$ and we need to predict the liabilities that correspond to the random variables $X_{i, j+1}$ for $(i, j+1) \in D_{t}$ where

$$
D_{t}=\{(i, j+1): i=t-J+1, \ldots, I, j=t-i, \ldots, J-1\}
$$

or equivalently to the random variables $\xi_{i, j+1}$ for $(i, j+1) \in D_{t}$. The following proposition gives the update formulas for the parameters of the posterior distribution of $\boldsymbol{\Phi}$ and derives the distributions of the random variables $\xi_{i, j+1},(i, j+1) \in D_{t}$, given $\mathcal{T}_{t}$.

Proposition 1 Given $\mathcal{T}_{t}$, for $j=0, \ldots, J-1$, the $\Phi_{j}$ are independent normally distributed random variables such that

$$
\left.\Phi_{j}\right|_{\mathcal{T}_{t}} \sim \mathcal{N}\left(\phi_{j}^{(t)},\left(s_{j}^{(t)}\right)^{2}\right)
$$

with posterior parameters

$$
\phi_{j}^{(t)}=\left(s_{j}^{(t)}\right)^{2}\left[\frac{\phi_{j}}{s_{j}^{2}}+\frac{1}{\sigma_{j}^{2}} \sum_{i=1}^{(t-j-1) \wedge I} \xi_{i, j+1}\right], \quad\left(s_{j}^{(t)}\right)^{2}=\left(\frac{1}{s_{j}^{2}}+\frac{(t-j-1) \wedge I}{\sigma_{j}^{2}}\right)^{-1} .
$$

Given $\mathcal{T}_{t},\left(\xi_{i, j+1}\right)_{(i, j+1) \in D_{t}}$ is a multivariate normally distributed random vector with

$$
\begin{aligned}
\mathbb{E}\left[\xi_{i, j+1} \mid \mathcal{T}_{t}\right] & =\phi_{j}^{(t)}, \quad(i, j+1) \in D_{t} \\
\operatorname{Cov}\left(\xi_{i, j+1}, \xi_{k, l+1} \mid \mathcal{T}_{t}\right) & =\mathbb{I}_{\{j=l\}}\left(\left(s_{j}^{(t)}\right)^{2}+\mathbb{I}_{\{i=k\}} \sigma_{j}^{2}\right), \quad(i, j+1) \times(k, l+1) \in D_{t}^{2} .
\end{aligned}
$$


In practice, we update at each time the posterior parameters according to the information generated by the new observations. Let

$$
\alpha_{j}^{(t+1)}=\frac{\left(s_{j}^{(t+1)}\right)^{2}}{\sigma_{j}^{2}}, \quad \beta_{j}^{(t+1)}=1-\alpha_{j}^{(t+1)}=\frac{\left(s_{j}^{(t+1)}\right)^{2}}{\left(s_{j}^{(t)}\right)^{2}}, \quad \gamma_{j}^{(t+q: t+l)}=\prod_{i=t+q}^{t+l} \beta_{j}^{(i)},
$$

with $\gamma_{j}^{(t+q: t+l)}=1$ if $q>l$. The following proposition gives the recursive update formulas from which we can deduce that, for $j=0, \ldots, J-1,\left(\phi_{j}^{(t)}\right)_{t=I, \ldots, I+j}$ is a $\mathcal{T}_{t}$-martingale.

Proposition 2 We have, for $t-j \leq I$,

$$
\phi_{j}^{(t+1)}=\beta_{j}^{(t+1)} \phi_{j}^{(t)}+\alpha_{j}^{(t+1)} \xi_{t-j, j+1}
$$

and, for $l=1, \ldots, 1+I+j-t$,

$$
\phi_{j}^{(t+l)}=\phi_{j}^{(t)} \gamma_{j}^{(t+1: t+l)}+\sum_{q=1}^{l} \gamma_{j}^{(t+q+1: t+l)} \alpha_{j}^{(t+q)} \xi_{t-j+q-1, j+1} .
$$

It follows that $\left(\phi_{j}^{(t)}\right)_{t=I, \ldots, I+j}$ is a $\mathcal{T}_{t}$-martingale, i.e. $\mathbb{E}\left[\phi_{j}^{(t+l)} \mid \mathcal{T}_{t}\right]=\phi_{j}^{(t)}$. Moreover

$$
\begin{aligned}
\log \left(\mathbb{E}\left[e^{\phi_{j}^{(t+l)}} \mid \mathcal{T}_{t}\right]\right)= & \phi_{j}^{(t)}+0.5\left(\left(s_{j}^{(t)}\right)^{2}+\sigma_{j}^{2}\right) \sum_{q=1}^{l}\left(\gamma_{j}^{(t+q+1: t+l)} \alpha_{j}^{(t+q)}\right)^{2} \\
& +\left(s_{j}^{(t)}\right)^{2} \sum_{1 \leq p<q \leq l} \gamma_{j}^{(t+p+1: t+l)} \gamma_{j}^{(t+q+1: t+l)} \alpha_{j}^{(t+p)} \alpha_{j}^{(t+q)} .
\end{aligned}
$$

Solvency II requires from non-life insurance companies a market-consistent valuation of their insurance liabilities. This implies that the outstanding loss liability cash flows need to be discounted with time values and should be determined given the latest information available. In old accounting tradition, insurance companies have estimated nominal (i.e. not discounted) claims reserves for their outstanding loss liabilities because undiscounting includes in fact a certain risk margin (depending on the level of the discount rate).

We define the undiscounted reserve at time $t$ for accident year $i=t-J+1, \ldots, I$ by

$$
R_{i, t}=\sum_{j=t-i+1}^{J} X_{i, j}=C_{i, J}-C_{i, t-i}
$$

and the discounted reserve by

$$
R_{i, t}^{(d)}=\sum_{j=t-i+1}^{J} \frac{X_{i, j}}{(1+r)^{j-t+i}}=\sum_{j=t-i+1}^{J} \frac{C_{i, j}-C_{i, j-1}}{(1+r)^{j-t+i}} .
$$

We restrict ourselves to a constant discount rate case for the sake of avoiding unnecessary complications but the case of a family of forward rates could also have been considered.

Let

$$
M_{l: m}^{(t)}=\sum_{j=l}^{m} \phi_{j}^{(t)} \quad \text { and } \quad S_{l: m}^{(t)}=\sum_{j=l}^{m}\left(\left(s_{j}^{(t)}\right)^{2}+\sigma_{j}^{2}\right)
$$

with the convention that $M_{l: m}^{(t)}=0$ and $S_{l: m}^{(t)}=0$ if $l>m$. The Best-Estimate outstanding loss Liabilities (BEL) are given in the following proposition. 
Proposition 3 For $i=t-J+1, \ldots, I$,

$$
\begin{aligned}
B E L_{i, t} & =\mathbb{E}\left[R_{i, t} \mid \mathcal{T}_{t}\right]=C_{i, t-i}\left(e^{\left.M_{t-i: J-1}^{(t)}+0.5 S_{t-i: J-1}^{(t)}-1\right)}\right. \\
B E L_{i, t}^{(d)} & =\mathbb{E}\left[R_{i, t}^{(d)} \mid \mathcal{T}_{t}\right]=C_{i, t-i}\left(\sum_{j=t-i}^{J-1} \frac{1}{(1+r)^{j-t+i+1}}\left(e^{M_{t-i: j}^{(t)}+0.5 S_{t-i: j}^{(t)}}-e^{M_{t-i: j-1}^{(t)}+0.5 S_{t-i: j-1}^{(t)}}\right)\right) .
\end{aligned}
$$

The undiscounted one year loss deterioration describes the deterioration of the expected reserves over the next year. It is defined at time $t$ for accident year $i=t-J+1, \ldots, I$ by

$$
L_{i, t}^{(1)}=X_{i, t+1-i}+B E L_{i, t+1}-B E L_{i, t}=\mathbb{E}\left[C_{i, J} \mid \mathcal{T}_{t+1}\right]-\mathbb{E}\left[C_{i, J} \mid \mathcal{T}_{t}\right] .
$$

The discounted one year loss deterioration is defined by

$$
L_{i, t}^{(1, d)}=\frac{X_{i, t+1-i}}{1+r}+\frac{1}{1+r} B E L_{i, t+1}^{(d)}-B E L_{i, t}^{(d)} .
$$

The one year deteriorations are centered, i.e. $\mathbb{E}\left[L_{i, t}^{(1)} \mid \mathcal{T}_{t}\right]=0$ and $\mathbb{E}\left[L_{i, t}^{(1, d)} \mid \mathcal{T}_{t}\right]=0$. Note that the undiscounted claims development results $(C R D)$ introduced by [11] are just the opposite of the undiscounted one year deteriorations.

\section{Approximation for quantiles of sums of log-normal random vari- ables}

Many problems in finance, insurance and engineering involve the evaluation of the distribution function of a random variable $S$ of the form

$$
S=\sum_{i=1}^{n} \delta_{i} e^{Z_{i}}
$$

where the $\delta_{i}$ are non-negative real numbers and $\left(Z_{1}, \ldots, Z_{n}\right)$ is a multivariate normal distributed random vector. In this paper we are mainly interested in the quantiles of $S$. Because it is impossible to obtain analytical expressions for the distribution function, Kaas, Dhaene and Goovaerts [10] propose to approximate $S$ by

$$
S^{l}=\mathbb{E}[S \mid \Lambda]
$$

for an appropriate choice of the conditioning $\Lambda$. The multi-dimensionality of the problem, caused by $\left(Z_{1}, Z_{2}, \ldots, Z_{n}\right)$, is then transformed to a single dimension leading to the comonotonicity of the vector $\left(\mathbb{E}\left[e^{Z_{1}} \mid \Lambda\right], \ldots, \mathbb{E}\left[e^{Z_{n}} \mid \Lambda\right]\right)$. From Jensen's inequality one can prove that $S^{l}$ is smaller in convex order than $S$. In literature a convex upper bound for $S$ has also been proposed (see e.g. [5] and $[6])$.

To get accurate approximations, $\Lambda$ should be chosen such that it is close to $S$. Consider the conditioning random variable $\Lambda$ as the linear combination of $Z_{1}, Z_{2}, \ldots, Z_{n}$ determined by

$$
\Lambda=\sum_{i=1}^{n} \gamma_{i} Z_{i}
$$

Then $S^{l}$ can be written

$$
S^{l}=\sum_{i=1}^{n} \delta_{i} e^{\mathbb{E}\left[Z_{i}\right]+0.5\left(1-r_{i}^{2}\right) \sigma_{Z_{i}}^{2}+r_{i} \sigma_{Z_{i}}(\Lambda-\mathbb{E}[\Lambda]) / \sigma_{\Lambda}}
$$


where $\sigma_{Z_{i}}^{2}$ and $\sigma_{\Lambda}^{2}$ are respectively the variances of $Z_{i}$ and $\Lambda$, and

$$
r_{i}=r_{i}\left(\gamma_{1}, \ldots, \gamma_{n}\right)=\frac{\operatorname{cov}\left(Z_{i}, \Lambda\right)}{\sigma_{Z_{i}} \sigma_{\Lambda}}
$$

Note that the expected values of the random variables $S$ and $S_{l}$ are equal and that, in case all $r_{i} \geq 0$,

$$
q_{99.5 \%}\left(S^{l}\right)=\sum_{i=1}^{n} \delta_{i} e^{\mathbb{E}\left[Z_{i}\right]+0.5\left(1-r_{i}^{2}\right) \sigma_{Z_{i}}^{2}+r_{i} \sigma_{Z_{i}} \varphi}
$$

where $\varphi$ is the VaR at the $99.5 \%$ level of the standard Gaussian distribution.

In [15],Vanduffel, Hoedemakers and Dhaene propose to choose $\Lambda$ such that a first-order approximation of the variance of $S^{l}$ is as large as possible and therefore the closest to the variance of $S$. The choices of the parameters $\gamma_{i}$ are then given by

$$
\gamma_{i}=\delta_{i} e^{\mathbb{E}\left[Z_{i}\right]+0.5 \sigma_{Z_{i}}^{2}}
$$

In [16], Vanduffel, Chen, Dhaene, Goovaerts, Henrard Kaas alternately propose to choose $\Lambda$ such that a first-order approximation of the $p$-level Conditional Tail Expectation of $S^{l}$ is as large as possible and therefore the closest to the $p$-level Conditional Tail Expectation of $S$. The choices of the parameters $\gamma_{i}$ are then given by

$$
\gamma_{i}=\delta_{i} e^{\mathbb{E}\left[Z_{i}\right]+0.5 \sigma_{Z_{i}}^{2}} \Phi^{\prime}\left(r_{i}\left(\delta_{1} e^{\mathbb{E}\left[Z_{1}\right]+0.5 \sigma_{Z_{1}}^{2}}, \ldots, \delta_{n} e^{\mathbb{E}\left[Z_{n}\right]+0.5 \sigma_{Z_{n}}^{2}}\right) \sigma_{Z_{i}}-\Phi^{-1}(p)\right)
$$

where $\Phi$ is the probability distribution function of a standard Gaussian random variable. As one can see, the parameters only differ up to proportional coefficients from the parameters that maximize the first-order approximation of the variance of $S^{l}$.

Since Monte-carlo simulations in [15] show that the approach by Vanduffel, Hoedemakers and Dhaene gives very accurate approximations for the 0.995-quantile of $S$, we keep their choice for the parameters $\gamma_{i}$ in the remainder of the paper.

\section{$4 \quad$ MVM for no discounted cash-flows}

We first consider the simple case where the cash flows are not discounted, although it is not coherent with the Solvency II framework. The case of discounted cash flows is provided in the next section.

The Fair-Value of Liabilities for accident year $i$ at time $t$ is defined as the sum of the Best Estimate valuation of Liabilities (BEL) and the Market Value Margin (MVM) of this accident year

$$
F V L_{i, t}=B E L_{i, t}+M V M_{i, t} .
$$

Let us remind that the Solvency Capital Requirement (SCR) is defined as the amount of capital required to support the claims paid out and the increase in the FVL following a one-in-200-year event over the next year, and that the Market Value Margin is defined as the sum of the current

and future SCRs. For accident year $i$ at time $t$, the SCR and the MVM are then given by the two simultaneous equations

$$
\begin{aligned}
S C R_{i, t} & =q_{99.5 \%}\left(F V L_{i, t+1}-F V L_{i, t}+X_{i, t+1-i} \mid \mathcal{T}_{t}\right) \\
& =q_{99.5 \%}\left(M V M_{i, t+1}-M V M_{i, t}+L_{i, t}^{(1)} \mid \mathcal{T}_{t}\right) \\
M V M_{i, t} & =c \sum_{j=t}^{i+J-1} \mathbb{E}\left[S C R_{i, j} \mid \mathcal{T}_{t}\right]
\end{aligned}
$$


where $c$ is the cost of capital rate (taken as a fixed $6 \%$ ). Note that $S C R_{i, t}$ and $M V M_{i, t}$ are $\mathcal{T}_{t^{-}}$ measurable random variables and that $S C R_{i, i+J}=0$ since all liabilities are completely extinct for this accident year after the accounting year $i+J: C_{i, J}=C_{i, J+1}=C_{i, J+2}=\ldots$

The Fair-Value of Liabilities for all accident years with outstanding liabilities is given by

$$
F V L_{t}=\sum_{i=t-J+1}^{I} B E L_{i, t}+M V M_{t}
$$

The $S C R_{t}$ and the $M V M_{t}$ are then given by the two simultaneous equations

$$
\begin{aligned}
S C R_{t} & =q_{99.5 \%}\left(F V L_{t+1}-F V L_{t}+\sum_{i=t-J+1}^{I} X_{i, t+1-i} \mid \mathcal{T}_{t}\right) \\
& =q_{99.5 \%}\left(M V M_{t+1}-M V M_{t}+L_{t}^{(1)} \mid \mathcal{T}_{t}\right) \\
M V M_{t} & =c \sum_{j=t}^{I+J-1} \mathbb{E}\left[S C R_{j} \mid \mathcal{T}_{t}\right]
\end{aligned}
$$

where

$$
L_{t}^{(1)}=\mathbb{E}\left[\sum_{i=1}^{I} C_{i, J} \mid \mathcal{T}_{t+1}\right]-\mathbb{E}\left[\sum_{i=1}^{I} C_{i, J} \mid \mathcal{T}_{t}\right] .
$$

Note that there is no reason $M V M_{t}$ and $S C R_{t}$ are respectively equal to $\sum_{i=t-J+1}^{I} M V M_{i, t}$ and $\sum_{i=t-J+1}^{I} S C R_{i, t}$ because the quantile function is nonlinear. Moreover $S C R_{I+J}=0$.

Throughout this section, we will use the following notation: for $I \leq t<l \leq i+J$,

$$
\begin{aligned}
\log \left(\sum_{i, l, t}\right)= & \frac{1}{2} \sum_{j=t-i}^{l-i-1}\left(\left(s_{j}^{(t)}\right)^{2}+\sigma_{j}^{2}\right)+\frac{1}{2} \sum_{j=l-i}^{J-1}\left(\left(s_{j}^{(t)}\right)^{2}+\sigma_{j}^{2}\right) \sum_{q=1}^{l-t}\left(\gamma_{j}^{(t+q+1: l)} \alpha_{j}^{(t+q)}\right)^{2} \\
& +\sum_{j=l-i}^{J-1}\left(s_{j}^{(t)}\right)^{2} \sum_{1 \leq p<q \leq l-t} \gamma_{j}^{(t+p+1: l)} \gamma_{j}^{(t+q+1: l)} \alpha_{j}^{(t+p)} \alpha_{j}^{(t+q)}
\end{aligned}
$$

and 0 otherwise.

\subsection{MVM for one accident year}

By using (4.6) and (4.7), the SCR expression for accident year $i$ can be developed as follows

$$
\begin{aligned}
S C R_{i, t} & =q_{99.5 \%}\left(c \sum_{j=t+1}^{i+J-1} \mathbb{E}\left[S C R_{i, j} \mid \mathcal{T}_{t+1}\right]-c \sum_{j=t}^{i+J-1} \mathbb{E}\left[S C R_{i, j} \mid \mathcal{T}_{t}\right]+\mathbb{E}\left[C_{i, J} \mid \mathcal{T}_{t+1}\right]-\mathbb{E}\left[C_{i, J} \mid \mathcal{T}_{t}\right] \mid \mathcal{T}_{t}\right) \\
& =q_{99.5 \%}\left(c \sum_{j=t+1}^{i+J-1} \mathbb{E}\left[S C R_{i, j} \mid \mathcal{T}_{t+1}\right]+\mathbb{E}\left[C_{i, J} \mid \mathcal{T}_{t+1}\right] \mid \mathcal{T}_{t}\right)-c \sum_{j=t}^{i+J-1} \mathbb{E}\left[S C R_{i, j} \mid \mathcal{T}_{t}\right]-\mathbb{E}\left[C_{i, J} \mid \mathcal{T}_{t}\right] .
\end{aligned}
$$

Since $\mathbb{E}\left[S C R_{i, t} \mid \mathcal{T}_{t}\right]=S C R_{i, t}$, we derive the recursive equations for the SCRs:

$$
S C R_{i, t}=\frac{1}{1+c}\left(q_{99.5 \%}\left(c \sum_{j=t+1}^{i+J-1} \mathbb{E}\left[S C R_{i, j} \mid \mathcal{T}_{t+1}\right]+\mathbb{E}\left[C_{i, J} \mid \mathcal{T}_{t+1}\right] \mid \mathcal{T}_{t}\right)-c \sum_{j=t+1}^{i+J-1} \mathbb{E}\left[S C R_{i, j} \mid \mathcal{T}_{t}\right]-\mathbb{E}\left[C_{i, J} \mid \mathcal{T}_{t}\right]\right)
$$


These equations have to be solved by starting at the final time period $(i+J-1)$ and working backward recursively. The following proposition gives the analytical recursive formulas for the SCR. The MVM is then deduced by using (4.7).

Proposition 4 For $t=I, \ldots, I+J$ and $i=t-J+1, \ldots, I$,

$$
S C R_{i, t}=C_{i, t-i} e^{M_{t-i: J-1}^{(t)}} a_{i, t}
$$

where, for $t \geq i+J, a_{i, t}=0$ and, for $t<i+J$,

$$
a_{i, t}=\frac{1}{1+c}\left[c \sum_{l=t+1}^{i+J-1} a_{i, l}\left(\Sigma_{i, l, t+1} \Xi_{i, t}-\Sigma_{i, l, t}\right)+e^{0.5 S_{t+1-i: J-1}^{(t+1)} \Xi_{i, t}}-e^{0.5 S_{t-i: J-1}^{(t)}}\right]
$$

with

$$
\log \left(\Xi_{i, t}\right)=\varphi \sqrt{\left(\left(s_{t-i}^{(t)}\right)^{2}+\sigma_{t-i}^{2}\right)+\sum_{j=t+1-i}^{J-1}\left(\alpha_{j}^{(t+1)}\right)^{2}\left(\left(s_{j}^{(t)}\right)^{2}+\sigma_{j}^{2}\right)} .
$$

Moreover, at the current date $t=I$, we have

$$
M V M_{i, I}=c C_{i, I-i} e^{M_{I-i: J-1}^{(I)}}\left[\sum_{l=I}^{i+J-1} a_{i, l} \Sigma_{i, l, I}\right] .
$$

It is worth noting that, given $C_{i, t-i}$ and $M_{t-i: J-1}^{(t)}=\sum_{j=t-i}^{J-1} \phi_{j}^{(t)}$, the sum of the posterior expected values of the random variables $\Phi_{j}$ given $\mathcal{T}_{t}$, the expressions for $S C R_{i, t}$ and $M V M_{i, t}$ are obtained by only calculating recursively the constants $a_{i, l}$ and $\Sigma_{i, l, t}$ for $l=t, \ldots, i+J-1$. In contrast to Bonnard, Daya and Margetts' results, the parameters of the log-normal distributions have to be updated given the latest information available.

\subsection{MVM for aggregated accident years}

In the previous subsection we have studied the MVM for one single accident year $i$. But in practice we want to calculate the MVM for all insurance liabilities, i.e. over all accident years. By using the same arguments as previously, we derive the recursive equations for the SCRs for all accident years: for $t=I, \ldots, I+J-1$,

$$
\begin{aligned}
S C R_{t}= & \frac{1}{1+c} q_{99.5 \%}\left(c \sum_{j=t+1}^{n-1} \mathbb{E}\left[S C R_{j} \mid \mathcal{T}_{t+1}\right]+\mathbb{E}\left[\sum_{i=1}^{I} C_{i, J} \mid \mathcal{T}_{t+1}\right] \mid \mathcal{T}_{t}\right) \\
& -\frac{1}{1+c}\left(c \sum_{j=t+1}^{n-1} \mathbb{E}\left[S C R_{j} \mid \mathcal{I}_{t}\right]+\mathbb{E}\left[\sum_{i=1}^{I} C_{i, J} \mid \mathcal{T}_{t}\right]\right) .
\end{aligned}
$$

The MVM is then given by

$$
M V M_{t}=c \sum_{j=t}^{I+J-1} \mathbb{E}\left[S C R_{j} \mid \mathcal{T}_{t}\right]
$$

Contrary to the previous case, there is no exact analytical recursive formula for the SCR because the SCRs are quantiles of sums of log-normal random variables over the different accident years. 
We need to approximate these quantiles such that they are equal to the sum of quantiles of some log-normal random variables but we have to choose these random variables in such a way that we are able to construct analytical recursive formulas for the SCRs.

Let us first introduce some notation. Let, for $i=t-J+1, \ldots, I$,

$$
r_{i}^{(t)}=\frac{1}{\varsigma_{i}^{(t)} \varsigma_{S}^{(t)}}\left(\sum_{l=t-J+1}^{i-1} \eta_{l}^{(t)}\left(\varsigma_{l}^{(t)}\right)^{2}+\left(\varsigma_{i}^{(t)}\right)^{2} \sum_{l=i}^{I} \eta_{l}^{(t)}\right)
$$

where

$$
\begin{aligned}
\left(\varsigma_{i}^{(t)}\right)^{2} & =\left(\left(s_{t-i}^{(t)}\right)^{2}+\sigma_{t-i}^{2}\right)+\sum_{j=t+1-i}^{J-1}\left(\alpha_{j}^{(t+1)}\right)^{2}\left(\left(s_{j}^{(t)}\right)^{2}+\sigma_{j}^{2}\right) \\
\left(\varsigma_{S}^{(t)}\right)^{2} & =\sum_{i=t-J+1}^{I}\left(\eta_{i}^{(t)}\right)^{2}\left(\varsigma_{i}^{(t)}\right)^{2}+2 \sum_{t-J+1 \leq i<l \leq I} \eta_{i}^{(t)} \eta_{l}^{(t)}\left(\varsigma_{i}^{(t)}\right)^{2}
\end{aligned}
$$

and

$$
\begin{aligned}
\log \left(\eta_{i}^{(t)}\right)= & \log \left(B_{i, t}^{(2)}\right)+\log \left(C_{i, I-i}\right)+M_{I-i: t-i-1}^{(I)}+0.5 S_{I-i: t-i-1}^{(I)} \\
& +\sum_{j=t+1-i}^{J-1} \beta_{j}^{(t+1)}\left[\phi_{j}^{(I)}+0.5 \beta_{j}^{(t+1)}\left(\left(s_{j}^{(I)}\right)^{2}+\sigma_{j}^{2}\right) \sum_{q=1}^{t-I}\left(\gamma_{j}^{(I+q+1: t)} \alpha_{j}^{(I+q)}\right)^{2}\right] \\
& +\sum_{j=t+1-i}^{J-1}\left(\beta_{j}^{(t+1)}\right)^{2}\left(s_{j}^{(I)}\right)^{2} \sum_{1 \leq p<q \leq t-I} \gamma_{j}^{(I+q+1: t)} \alpha_{j}^{(I+q)} \gamma_{j}^{(I+p+1: t)} \alpha_{j}^{(I+p)} \\
& +\left(\phi_{t-i}^{(I)}+\sum_{j=t+1-i}^{J-1} \alpha_{j}^{(t+1)} \phi_{j}^{(I)}\right)+0.5\left(\left(\left(s_{t-i}^{(I)}\right)^{2}+\sigma_{t-i}^{2}\right)+\sum_{j=t+1-i}^{J-1}\left(\alpha_{j}^{(t+1)}\right)^{2}\left(\left(s_{j}^{(I)}\right)^{2}+\sigma_{j}^{2}\right)\right)
\end{aligned}
$$

with

$$
B_{i, t}^{(2)}=\sum_{l=t+1}^{i+J-1} c b_{i, l} \Sigma_{i, l, t+1}+e^{0.5 S_{t+1-i: J-1}^{(t+1)}}
$$

and $b_{i, l}$ defined in (4.13). The parameters $r_{i}^{(t)}, \eta_{i}^{(t)},\left(\varsigma_{i}^{(t)}\right)^{2}$ and $\left(\varsigma_{S}^{(t)}\right)^{2}$ are respectively the equivalents of the correlation coefficients $r_{i}$ in (3.3), the constants $\gamma_{i}$ in (3.4), the variances $\sigma_{Z_{i}}^{2}$ and $\sigma_{\Lambda}^{2}$ of $Z_{i}$ and $\Lambda$ in Section 3.

The following proposition gives the recursive approximated formulas and derive the approximated expression for the MVM.

Proposition 5 For $t=I, \ldots, I+J$,

$$
S C R_{t} \simeq \sum_{i=t-J+1}^{I} C_{i, t-i} e^{M_{t-i: J-1}^{(t)}} b_{i, t}
$$

where $b_{i, t}$ is defined recursively by

- for $t \geq i+J, b_{i, t}=0$,

- for $t<i+J$

$$
b_{i, t}=\frac{1}{1+c}\left[c \sum_{l=t+1}^{i+J-1} b_{i, l}\left(\Sigma_{i, l, t+1} \Xi_{i, t}^{S}-\Sigma_{i, l, t}\right)+e^{0.5 S_{t+1-i: J-1}^{(t+1)} \Xi_{i, t}^{S}}-e^{0.5 S_{t-i: J-1}^{(t)}}\right]
$$


with

$$
\log \left(\Xi_{i, t}^{S}\right)=0.5\left(1-\left(r_{i}^{(t)}\right)^{2}\right)\left(\varsigma_{i}^{(t)}\right)^{2}+r_{i}^{(t)} \varsigma_{i}^{(t)} \varphi
$$

Moreover, at the current date $t=I$, we have

$$
M V M_{I} \simeq c \sum_{l=I}^{I+J-1} \sum_{i=l-J+1}^{I} C_{i, I-i} b_{i, l} \Sigma_{i, l, I} e^{M_{I-i: J-1}^{(I)}} .
$$

It can be seen that $\Xi_{i, t}^{S} \neq \Xi_{i, t}$ even if the losses of the accident years are independent. It follows that $b_{i, t} \neq a_{i, t}$ and that $M V M_{I} \neq \sum_{i=I-J+1}^{I} M V M_{i, I}$.

\section{$5 \quad$ MVM for discounted cash-flows}

We now consider the case where the cash flows are discounted for the calculations of the reserves, the SCRs and the MVM, following the Solvency II framework.

The Fair-Value of Liabilities for accident year $i$ at time $t$ is still defined as the sum of the Best Estimate valuation of Liabilities $\left(B E L_{i, t}^{(d)}\right)$ and the Market Value Margin $\left(M V M_{i, t}^{(d)}\right)$ of this accident year, but the $S C R_{i, t}^{(d)}$ and the $M V M_{i, t}^{(d)}$ are now given by the two simultaneous equations

$$
\begin{aligned}
S C R_{i, t}^{(d)} & =q_{99.5 \%}\left(\frac{1}{1+r} M V M_{i, t+1}^{(d)}-M V M_{i, t}^{(d)}+L_{i, t}^{(1, d)} \mid \mathcal{T}_{t}\right) \\
M V M_{i, t} & =c \sum_{j=t}^{i+J-1} \mathbb{E}\left[\frac{S C R_{i, j}^{(d)}}{(1+r)^{j-t}} \mid \mathcal{T}_{t}\right] .
\end{aligned}
$$

Note that $S C R_{i, i+J}^{(d)}$ is still null.

The $S C R_{t}^{(d)}$ and the $M V M_{t}^{(d)}$ for all accident years are defined by the two simultaneous equations

$$
\begin{aligned}
S C R_{t}^{(d)} & =q_{99.5 \%}\left(\frac{1}{1+r} M V M_{t+1}^{(d)}-M V M_{t}^{(d)}+L_{t}^{(1, d)} \mid \mathcal{T}_{t}\right) \\
M V M_{t} & =c \sum_{j=t}^{I+J-1} \mathbb{E}\left[\frac{S C R_{j}^{(d)}}{(1+r)^{j-t}} \mid \mathcal{T}_{t}\right]
\end{aligned}
$$

where

$$
\begin{aligned}
L_{t}^{(1, d)} & =\sum_{i=t-J+1}^{I} L_{i, t}^{(1, d)}=\sum_{i=t-J+1}^{I}\left(\frac{X_{i, t+1-i}}{1+r}+\frac{1}{1+r} B E L_{i, t+1}^{(d)}-B E L_{i, t}^{(d)}\right) \\
& =\sum_{i=t-J+1}^{I}\left(\frac{X_{i, t+1-i}}{1+r}+\frac{1}{1+r} \mathbb{E}\left[\sum_{j=t+2}^{i+J} \frac{X_{i, j-i}}{(1+r)^{j-t-1}} \mid \mathcal{T}_{t+1}\right]-\mathbb{E}\left[\sum_{j=t+1}^{i+J} \frac{X_{i, j-i}}{(1+r)^{j-t}} \mid \mathcal{T}_{t}\right]\right) .
\end{aligned}
$$

Note that $S C R_{I+J}^{(d)}=0$.

Throughout this section, we will use the following notation: for $I \leq t<l \leq i+j$,

$$
\begin{aligned}
\log \left(\Sigma_{i, j, l, t}\right)= & \frac{1}{2} \sum_{k=t-i}^{l-i-1}\left(\left(s_{k}^{(t)}\right)^{2}+\sigma_{k}^{2}\right)+\frac{1}{2} \sum_{k=l-i}^{j}\left(\left(s_{k}^{(t)}\right)^{2}+\sigma_{k}^{2}\right) \sum_{q=1}^{l-t}\left(\gamma_{k}^{(t+q+1: l)} \alpha_{k}^{(t+q)}\right)^{2} \\
& +\sum_{k=l-i}^{j}\left(s_{k}^{(t)}\right)^{2} \sum_{1 \leq p<q \leq l-t} \gamma_{k}^{(t+p+1: l)} \gamma_{k}^{(t+q+1: l)} \alpha_{k}^{(t+p)} \alpha_{k}^{(t+q)}
\end{aligned}
$$


and 0 otherwise.

\subsection{MVM for one accident year}

By using (5.14) and (5.15), and since

$$
L_{i, t}^{(1, d)}=\frac{X_{i, t+1-i}}{1+r}+\frac{1}{1+r} \mathbb{E}\left[\sum_{j=t+2}^{i+J} \frac{X_{i, j-i}}{(1+r)^{j-t-1}} \mid \mathcal{T}_{t+1}\right]-\mathbb{E}\left[\sum_{j=t+1}^{i+J} \frac{X_{i, j-i}}{(1+r)^{j-t}} \mid \mathcal{T}_{t}\right]
$$

the SCR expression for accident year $i$ can be developed as follows

$$
\begin{aligned}
S C R_{i, t}^{(d)}= & q_{99.5 \%}\left(c \sum_{j=t+1}^{i+J-1} \mathbb{E}\left[\frac{S C R_{i, j}^{(d)}}{(1+r)^{j-t}} \mid \mathcal{T}_{t+1}\right]+\frac{X_{i, t+1-i}}{1+r}+\frac{1}{1+r} \mathbb{E}\left[\sum_{j=t+2}^{i+J} \frac{X_{i, j-i}}{(1+r)^{j-t-1}} \mid \mathcal{T}_{t+1}\right] \mid \mathcal{T}_{t}\right) \\
& -c \sum_{j=t}^{i+J-1} \mathbb{E}\left[\frac{S C R_{i, j}^{(d)}}{(1+r)^{j-t}} \mid \mathcal{T}_{t}\right]-\mathbb{E}\left[\sum_{j=t+1}^{i+J} \frac{X_{i, j-i}}{(1+r)^{j-t}} \mid \mathcal{T}_{t}\right] .
\end{aligned}
$$

Since $\mathbb{E}\left[S C R_{i, t}^{(d)} \mid \mathcal{T}_{t}\right]=S C R_{i, t}^{(d)}$, we derive the recursive equations for the SCRs: for $t \leq i+J-1$

$$
\begin{aligned}
S C R_{i, t}^{(d)}= & \frac{1}{1+c}\left(q_{99.5 \%}\left(c \sum_{j=t+1}^{i+J-1} \mathbb{E}\left[\frac{S C R_{i, j}^{(d)}}{(1+r)^{j-t}} \mid \mathcal{T}_{t+1}\right]+\frac{X_{i, t+1-i}}{1+r}+\mathbb{E}\left[\sum_{j=t+2}^{i+J} \frac{X_{i, j-i}}{(1+r)^{j-t}} \mid \mathcal{T}_{t+1}\right] \mid \mathcal{T}_{t}\right) .1 \mathcal{Q}\right) \\
& -\frac{1}{1+c}\left(c \sum_{j=t+1}^{i+J-1} \mathbb{E}\left[\frac{S C R_{i, j}^{(d)}}{(1+r)^{j-t}} \mid \mathcal{T}_{t}\right]+\mathbb{E}\left[\sum_{j=t+1}^{i+J} \frac{X_{i, j-i}}{(1+r)^{j-t}} \mid \mathcal{T}_{t}\right]\right)
\end{aligned}
$$

These equations have to be solved by starting at the final time period $(i+J-1)$ and working backwards recursively. But it is necessary to approximate the quantiles of the sum of the SCRs and the current an future discounted cash flows in such a way that we are able to construct analytical recursive formulas for the SCRs.

Let us introduce some notation:

$$
r_{i, j}^{(t)}=\frac{1}{\varsigma_{i, j}^{(t)} \varsigma_{S, i}^{(t)}}\left(\sum_{l=t-i}^{j-1} \eta_{i, l}^{(t)}\left(\varsigma_{i, l}^{(t)}\right)^{2}+\left(\varsigma_{i, j}^{(t)}\right)^{2} \sum_{l=j}^{J-1} \eta_{i, l}^{(t)}\right)
$$

where

$$
\begin{aligned}
\left(\varsigma_{i, j}^{(t)}\right)^{2} & =\left(\left(s_{t-i}^{(t)}\right)^{2}+\sigma_{t-i}^{2}\right)+\sum_{l=t+1-i}^{j}\left(\alpha_{l}^{(t+1)}\right)^{2}\left(\left(s_{l}^{(t)}\right)^{2}+\sigma_{l}^{2}\right) \\
\left(\varsigma_{S, i}^{(t)}\right)^{2} & =\sum_{j=t-i}^{J-1}\left(\eta_{i, j}^{(t)}\right)^{2}\left(\varsigma_{i, j}^{(t)}\right)^{2}+2 \sum_{t-i \leq j<l \leq J-1} \eta_{i, j}^{(t)} \eta_{i, l}^{(t)}\left(\varsigma_{i, j}^{(t)}\right)^{2}
\end{aligned}
$$


and

$$
\begin{aligned}
\log \left(\eta_{i, j}^{(t)}\right)= & \log \left(A_{i, j, t}^{(2), d}\right)+\log \left(C_{i, I-i}\right)+M_{I-i: t-i-1}^{(I)}+0.5 S_{I-i: t-i-1}^{(I)} \\
& +\sum_{l=t+1-i}^{j} \beta_{l}^{(t+1)}\left[\phi_{l}^{(I)}+0.5 \beta_{l}^{(t+1)}\left(\left(s_{l}^{(I)}\right)^{2}+\sigma_{l}^{2}\right) \sum_{q=1}^{t-I}\left(\gamma_{l}^{(I+q+1: t)} \alpha_{l}^{(I+q)}\right)^{2}\right] \\
& +\sum_{l=t+1-i}^{j}\left(\beta_{l}^{(t+1)}\right)^{2}\left(s_{l}^{(I)}\right)^{2} \sum_{1 \leq p<q \leq t-I} \gamma_{l}^{(I+q+1: t)} \alpha_{l}^{(I+q)} \gamma_{l}^{(I+p+1: t)} \alpha_{l}^{(I+p)} \\
& +\left(\phi_{t-i}^{(I)}+\sum_{l=t+1-i}^{j} \alpha_{j}^{(t+1)} \phi_{l}^{(I)}\right)+0.5\left(\left(\left(s_{t-i}^{(I)}\right)^{2}+\sigma_{t-i}^{2}\right)+\sum_{l=t+1-i}^{j}\left(\alpha_{l}^{(t+1)}\right)^{2}\left(\left(s_{l}^{(I)}\right)^{2}+\sigma_{l}^{2}\right)\right)
\end{aligned}
$$

with $A_{i, j, t}^{(2), d}$ defined in the following proposition.

Proposition 6 For $i=t-J+1, \ldots, I$

$$
S C R_{i, t}^{(d)} \simeq C_{i, t-i} \sum_{j=t-i}^{J-1} e^{M_{t-i: j}^{(t)}} a_{i, j, t}
$$

where $a_{i, j, t}$ is defined recursively by

- for $j=I-i, \ldots, J-1$ and $t \geq i+j+1, a_{i, j, t}=0$

- for $j=t-i, \ldots, J-1$, we have

$$
a_{i, j, t}=\frac{1}{1+c}\left(A_{i, j, t}^{(2), d} \Xi_{i, j, t}^{(d)}-A_{i, j, t}^{(1), d}\right)
$$

where

$$
\begin{aligned}
A_{i, t-i, t}^{(1), d} & =\frac{r}{(1+r)^{2}} e^{0.5 S_{t-i: t-i}^{(t)}} \\
A_{i, j, t}^{(1), d} & =\sum_{l=t+1}^{i+j} \frac{c}{(1+r)^{l-t}} a_{i, j, l} \Sigma_{i, j, l, t}+\frac{r}{(1+r)^{j-t+i+2}} e^{0.5 S_{t-i: j}^{(t)}}, \quad j=t+1-i, \ldots, J-2 \\
A_{i, J-1, t}^{(1), d} & =\sum_{l=t+1}^{i+J-1} \frac{c}{(1+r)^{l-t}} a_{i, J-1, l} \Sigma_{i, j, l, t}+\frac{1}{(1+r)^{J-t+1+i}} e^{0.5 S_{t-i: J-1}^{(t)}}
\end{aligned}
$$

and

$$
\begin{aligned}
A_{i, t-i, t}^{(2), d} & =\frac{r}{(1+r)^{2}} \\
A_{i, j, t}^{(2), d} & =\left(\sum_{l=t+1}^{i+j} \frac{c}{(1+r)^{l-t}} a_{i, j, l} \Sigma_{i, j, l, t+1}+\frac{r}{(1+r)^{j-t+i+2}} e^{0.5 S_{t+1-i: j}^{(t+1)}}\right), \quad j=t+1-i, \ldots, J-2 \\
A_{i, J-1, t}^{(2), d} & =\left(\left[\sum_{l=t+1}^{i+J-1} \frac{c}{(1+r)^{l-t}} a_{i, J-1, l} \Sigma_{i, J-1, l, t+1}\right]+\frac{1}{(1+r)^{J-t+1+i}} e^{0.5 S_{t+1-i: J-1}^{(t+1)}}\right) .
\end{aligned}
$$

and

$$
\Xi_{i, j, t}^{(d)}=e^{0.5\left(1-\left(r_{i, j}^{(t)}\right)^{2}\right)\left(\varsigma_{i, j}^{(t)}\right)^{2}+r_{i, j}^{(t)} \varsigma_{i, j}^{(t)} \varphi} .
$$

Moreover, at the current date $t=I$, we have

$$
M V M_{i, I}^{(d)} \simeq c C_{i, I-i} \sum_{j=I-i}^{J-1} e^{M_{I-i: j}^{(I)}}\left[\sum_{l=I}^{i+j-1} a_{i, j, l} \Sigma_{i, j, l, I}\right] .
$$




\subsection{MVM for aggregated accident years}

For completeness sake, we give the MVM for all accident years. By using the same arguments as previously, we derive the recursive equations for the SCRs for all accident years: for $t=I, \ldots, I+$ $J-1$

$$
\begin{aligned}
& S C R_{t}^{(d)} \\
= & \frac{1}{1+c}\left(q_{99.5 \%}\left(c \sum_{j=t+1}^{I+J-1} \mathbb{E}\left[\frac{S C R_{j}^{(d)}}{(1+r)^{j-t}} \mid \mathcal{T}_{t+1}\right]+\sum_{i=t-J+1}^{I}\left(\frac{X_{i, t+1-i}}{1+r}+\mathbb{E}\left[\sum_{j=t+2}^{i+J} \frac{X_{i, j-i}}{(1+r)^{j-t}} \mid \mathcal{T}_{t+1}\right]\right) \mid \mathcal{T}_{t}\right)\right) \\
& -\frac{1}{1+c}\left(c \sum_{j=t+1}^{I+J-1} \mathbb{E}\left[\frac{S C R_{j}^{(d)}}{(1+r)^{j-t}} \mid \mathcal{T}_{t}\right]+\sum_{i=t-J+1}^{I} \mathbb{E}\left[\sum_{j=t+1}^{i+J} \frac{X_{i, j-i}}{(1+r)^{j-t}} \mid \mathcal{T}_{t}\right]\right) .
\end{aligned}
$$

Let

$$
\bar{r}_{i, j}^{(t)}=\frac{1}{\varsigma_{i, j}^{(t)} \varsigma_{\bar{S}}^{(t)}} \sum_{k=t-J+1}^{I} \sum_{l=t-k}^{J-1} \bar{\eta}_{k, l}^{(t)}\left(\varsigma_{i \wedge k, j \wedge l}^{(t)}\right)^{2}
$$

where

$$
\begin{aligned}
\left(\varsigma_{i, j}^{(t)}\right)^{2} & =\left(\left(s_{t-i}^{(t)}\right)^{2}+\sigma_{t-i}^{2}\right)+\sum_{l=t+1-i}^{j}\left(\alpha_{l}^{(t+1)}\right)^{2}\left(\left(s_{l}^{(t)}\right)^{2}+\sigma_{l}^{2}\right) \\
\left(\varsigma_{\bar{S}}^{(t)}\right)^{2} & =\sum_{t-J+1 \leq i, k \leq I} \sum_{j=t-i}^{J-1} \sum_{l=t-k}^{J-1} \bar{\eta}_{i, j}^{(t)} \bar{\eta}_{k, l}^{(t)}\left(\varsigma_{i \wedge k, j \wedge l}^{(t)}\right)^{2}
\end{aligned}
$$

and

$$
\begin{aligned}
\log \left(\bar{\eta}_{i, j}^{(t)}\right)= & \log \left(B_{i, j, t}^{(2), d}\right)+\log \left(C_{i, I-i}\right)+M_{I-i: t-i-1}^{(I)}+0.5 S_{I-i: t-i-1}^{(I)} \\
& +\sum_{l=t+1-i}^{j} \beta_{l}^{(t+1)}\left[\phi_{l}^{(I)}+0.5 \beta_{l}^{(t+1)}\left(\left(s_{l}^{(I)}\right)^{2}+\sigma_{l}^{2}\right) \sum_{q=1}^{t-I}\left(\gamma_{l}^{(I+q+1: t)} \alpha_{l}^{(I+q)}\right)^{2}\right] \\
& +\sum_{l=t+1-i}^{j}\left(\beta_{l}^{(t+1)}\right)^{2}\left(s_{l}^{(I)}\right)^{2} \sum_{1 \leq p<q \leq t-I} \gamma_{l}^{(I+q+1: t)} \alpha_{l}^{(I+q)} \gamma_{l}^{(I+p+1: t)} \alpha_{l}^{(I+p)} \\
& +\left(\phi_{t-i}^{(I)}+\sum_{l=t+1-i}^{j} \alpha_{j}^{(t+1)} \phi_{l}^{(I)}\right)+0.5\left(\left(\left(s_{t-i}^{(I)}\right)^{2}+\sigma_{t-i}^{2}\right)+\sum_{l=t+1-i}^{j}\left(\alpha_{l}^{(t+1)}\right)^{2}\left(\left(s_{l}^{(I)}\right)^{2}+\sigma_{l}^{2}\right)\right)
\end{aligned}
$$$$
\text { with } B_{i, j, t}^{(2), d} \text { defined in the following proposition. }
$$

Proposition 7 For $i=t-J+1, \ldots, I$

$$
S C R_{i, t}^{(d)} \simeq \sum_{i=t-J+1}^{I} C_{i, t-i} \sum_{j=t-i}^{J-1} e^{M_{t-i: j}^{(t)}} b_{i, j, t}
$$

where $b_{i, j, t}$ is defined recursively by

$$
\text { - for } j=I-i, \ldots, J-1 \text { and } t \geq i+j+1, b_{i, j, t}=0
$$


- for $j=t-i, \ldots, J-1$, we have

$$
b_{i, j, t}=\frac{1}{1+c}\left(B_{i, j, t}^{(2), d} \Xi_{i, j, t}^{S,(d)}-B_{i, j, t}^{(1), d}\right)
$$

where

$$
\begin{aligned}
B_{i, t-i, t}^{(1), d} & =\frac{r}{(1+r)^{2}} e^{0.5 S_{t-i: t-i}^{(t)}} \\
B_{i, j, t}^{(1), d} & =\sum_{l=t+1}^{i+j} \frac{c}{(1+r)^{l-t}} b_{i, j, l} \Sigma_{i, j, l, t}+\frac{r}{(1+r)^{j-t+i+2}} e^{0.5 S_{t-i: j}^{(t)}}, \quad j=t+1-i, \ldots, J-2 \\
B_{i, J-1, t}^{(1), d} & =\sum_{l=t+1}^{i+J-1} \frac{c}{(1+r)^{l-t}} b_{i, j, l} \Sigma_{i, j, l, t}+\frac{1}{(1+r)^{J-t+i}} e^{0.5 S_{t-i: J-1}^{(t)}}
\end{aligned}
$$

and

$$
\begin{aligned}
B_{i, t-i, t}^{(2), d} & =\frac{r}{(1+r)^{2}} \\
B_{i, j, t}^{(2), d} & =\left(\sum_{l=t+1}^{i+j} \frac{c}{(1+r)^{l-t}} b_{i, j, l} \Sigma_{i, j, l, t+1}+\frac{r}{(1+r)^{j-t+i+2}} e^{0.5 S_{t+1-i: j}^{(t+1)}}\right), \quad j=t+1-i, \ldots, J-2 \\
B_{i, J-1, t}^{(2), d} & =\left(\left[\sum_{l=t+1}^{i+J-1} \frac{c}{(1+r)^{l-t}} b_{i, j, l} \Sigma_{i, j, l, t+1}\right]+\frac{1}{(1+r)^{J-t+1+i}} e^{0.5 S_{l+1-i: J-1}^{(t+1)}}\right)
\end{aligned}
$$

and

$$
\Xi_{i, j, t}^{S,(d)}=e^{0.5\left(1-\left(\bar{r}_{i, j}^{(t)}\right)^{2}\right)\left(\varsigma_{i, j}^{(t)}\right)^{2}+\bar{r}_{i, j}^{(t)} \varsigma_{i, j}^{(t)} \varphi} .
$$

Moreover, at the current date $t=I$, we have

$$
M V M_{i, I}^{(d)} \simeq c \sum_{i=t-J+1}^{I} C_{i, I-i} \sum_{j=I-i}^{J-1} e^{M_{I-i: j}^{(t)}}\left[\sum_{l=I}^{i+j-1} b_{i, j, l} \Sigma_{i, j, l, I}\right] .
$$

\section{Case study}

We now present a case study. The data set is the $17 \times 17$ private liability insurance cash-flow triangle considered in [18]. In this paper, Wuthrich, Embrechts and Tsanakas use probability distortion methods to calculate risk margins. In Table 3 we provide the cumulative payments $C_{i, j}=\sum_{l=0}^{j} X_{i, l}$ of accident years $i=1, \ldots, 17$. We consider the run-off situation at time $I$ and assume that all claims are settled after development year $J=16$.

\begin{tabular}{|ccccccccc|}
\hline$j$ & 0 & 1 & 2 & 3 & 4 & 5 & 6 & 7 \\
\hline$\phi_{j}$ & $7.90 \mathrm{e}-02$ & $5.62 \mathrm{e}-02$ & $4.00 \mathrm{e}-02$ & $2.85 \mathrm{e}-02$ & $2.02 \mathrm{e}-02$ & $1.44 \mathrm{e}-02$ & $1.02 \mathrm{e}-02$ & $7.29 \mathrm{e}-03$ \\
$\sigma_{j}^{2}$ & $6.80 \mathrm{e}-04$ & $4.52 \mathrm{e}-04$ & $3.01 \mathrm{e}-04$ & $2.00 \mathrm{e}-04$ & $1.33 \mathrm{e}-04$ & $8.85 \mathrm{e}-05$ & $5.89 \mathrm{e}-05$ & $3.92 \mathrm{e}-05$ \\
$s_{j}^{2}$ & $1.00 \mathrm{e}-02$ & $7.36 \mathrm{e}-03$ & $5.41 \mathrm{e}-03$ & $3.98 \mathrm{e}-03$ & $2.93 \mathrm{e}-03$ & $2.15 \mathrm{e}-03$ & $1.58 \mathrm{e}-03$ & $1.17 \mathrm{e}-03$ \\
\hline$j$ & 8 & 9 & 10 & 11 & 12 & 13 & 14 & 15 \\
\hline$\phi_{j}$ & $5.18 \mathrm{e}-03$ & $3.69 \mathrm{e}-03$ & $2.62 \mathrm{e}-03$ & $1.87 \mathrm{e}-03$ & $1.33 \mathrm{e}-03$ & $9.45 \mathrm{e}-04$ & $6.72 \mathrm{e}-04$ & $4.78 \mathrm{e}-04$ \\
$\sigma_{j}^{2}$ & $2.61 \mathrm{e}-05$ & $1.73 \mathrm{e}-05$ & $1.15 \mathrm{e}-05$ & $7.67 \mathrm{e}-06$ & $5.10 \mathrm{e}-06$ & $3.39 \mathrm{e}-06$ & $2.26 \mathrm{e}-06$ & $1.50 \mathrm{e}-06$ \\
$s_{j}^{2}$ & $8.58 \mathrm{e}-04$ & $6.31 \mathrm{e}-04$ & $4.64 \mathrm{e}-04$ & $3.41 \mathrm{e}-04$ & $2.51 \mathrm{e}-04$ & $1.85 \mathrm{e}-04$ & $1.36 \mathrm{e}-04$ & $1.00 \mathrm{e}-04$ \\
\hline
\end{tabular}

Table 1: Prior parameters $\left(\phi_{j}, s_{j}^{2}\right)$ and standard deviation parameters $\sigma_{j}^{2}$ of the Bayesian log-normal chain ladder model 
The parameters of the Bayesian log-normal chain ladder model are provided in Table 1. Note that they differ from those given in [18] because the assumptions of the models are not the same. Wuthrich, Embrechts and Tsanakas consider a Bayesian chain ladder model where $\log \left(F_{i, j}-1\right)$ is normally distributed, assuming that the cash-flows of the triangle are necessarily positive.

Using these parameter choices and the liability insurance cash-flow triangle, we are able to evaluate the posterior parameters and then to calculate the BEL and the MVM for the run-off portfolio. The cost-of-capital rate is chosen to be $c=6 \%$. According to the previous sections, we compute these quantities when cash flows are not discounted or when they are subject to a constant discount rate $r$. Moreover to measure the diversification effect between accident years, we compare the sum $\sum_{i=t-J+1}^{I} M V M_{i, I}$ to $M V M_{I}$ and the sum $\sum_{i=t-J+1}^{I} M V M_{i, I}^{(d)}$ to $M V M_{I}^{(d)}$. Table 2 presents an overview of the numerical results.

\begin{tabular}{|c|ccc|c|ccc|}
\cline { 2 - 7 } \multicolumn{1}{c|}{} & \multicolumn{3}{c|}{ No discounted cash flows } & \multicolumn{4}{c|}{ Discounted cash flows } \\
\cline { 2 - 8 } \multicolumn{1}{c|}{} & BEL & MVM & BEL/MVM & $r$ & BEL & MVM & BEL/MVM \\
\hline \multirow{3}{*}{$\begin{array}{c}\text { Without } \\
\text { diversification }\end{array}$} & & & & & & & \\
& & & & 0.01 & 23198 & 1745 & $7.52 \%$ \\
& & & 0.02 & 22518 & 1696 & $7.52 \%$ \\
With & 23921 & 1398 & $5,84 \%$ & 0 & 23921 & 1398 & $5,84 \%$ \\
diversification & & & & 0.01 & 23198 & 1344 & $5,79 \%$ \\
& & & & 0.02 & 22518 & 1294 & $5,74 \%$ \\
& & & & 0.04 & 21278 & 1201 & $5,64 \%$ \\
\hline
\end{tabular}

Table 2: BEL and MVM for the data set given in Table 3 when cash-flows are discounted or not.

We first observe that our BEL is 3\% less than the best-estimate reserves given in [18]. Of course this value heavily depends on the choices of the model and of the parameters.

Let us begin with the case where cash flows are not discounted. We obtain a MVM of 1800 (which is $7.52 \%$ in terms of the BEL) when we sum the individual MVMs over all the accident years and 1398 (which is 5.84\% in terms of the BEL) when we compute the MVM for aggregated accident years. As expected, we find the first approach is more conservative than the second approach. Diversification effects between accident years account for substantial releases of over $24 \%$.

Let us now consider the case where cash flows are discounted. When $r=0$, we obtain the same values as in the previous case (which is logical). When $r$ increases, the BEL and the MVM decrease but approximately in the same way since the ratios BEL/MVM remain constant when $r$ is not too large. This can also be observed in [18].

Finally we see that our MVMs are larger than those found in [18] (approximately 20\% more when the risk aversion parameters are chosen by Wuthrich, Embrechts and Tsanakas such that the value of the risk margins are of the same order as those found with the approach from SalzmannWuthrich [14]). However, note that the question of the choice of the risk aversion parameters does not need to be raised in our case since we follow the MVM methodology as prescribed in the Solvency II framework. 


\section{Proofs}

\subsection{Proof of Proposition 1}

First note that we have

$$
\begin{aligned}
\mathcal{T}_{t} & =\sigma\left\{C_{i, j}: i+j \leq t, i=1, \ldots, I, j=0, \ldots, J\right\} \\
& =\sigma\left\{\xi_{i, j}, C_{i, 0}: i+j \leq t, i=1, \ldots, I, j=1, \ldots, J\right\} .
\end{aligned}
$$

The conditional distribution of $\boldsymbol{\Phi}$ satisfies the following proportionality properties

$$
\begin{aligned}
\pi\left(\boldsymbol{\Phi} \mid \mathcal{T}_{t}\right) & \propto \pi(\boldsymbol{\Phi}) \pi\left(\left(\xi_{i, l}, C_{i, 0}\right)_{i+l \leq t, i=1, \ldots, I, l=1, \ldots, J} \mid \boldsymbol{\Phi}\right) \\
& \propto \prod_{j=1}^{J-1} \pi\left(\Phi_{j}\right) \pi\left(\left(\xi_{i, j+1}, C_{i, 0}\right)_{i+j+1 \leq t, i=1, \ldots, I} \mid \Phi_{j}\right) \\
& \propto \prod_{j=1}^{J-1} \exp \left(-\frac{1}{2} \frac{\left(\Phi_{j}-\phi_{j}\right)^{2}}{s_{j}^{2}}\right) \prod_{i=1}^{(t-j-1) \wedge I} \exp \left(-\frac{1}{2} \frac{\left(\xi_{i, j+1}-\Phi_{j}\right)^{2}}{\sigma_{j}^{2}}\right) \\
& \propto \prod_{j=1}^{J-1} \exp \left(-\frac{1}{2}\left(\frac{\Phi_{j}^{2}-2 \phi_{j} \Phi_{j}}{s_{j}^{2}}+\sum_{i=1}^{(t-j-1) \wedge I} \frac{\Phi_{j}^{2}-2 \Phi_{j} \xi_{i, j+1}}{\sigma_{j}^{2}}\right)\right) \\
& \propto \prod_{j=1}^{J-1} \exp \left(-\frac{1}{2}\left(\Phi_{j}^{2}\left(\frac{1}{s_{j}^{2}}+\frac{(t-j-1) \wedge I}{\sigma_{j}^{2}}\right)-2 \Phi_{j}\left(\frac{\phi_{j}}{s_{j}^{2}}+\frac{1}{\sigma_{j}^{2}} \sum_{i=1}^{(t-j-1) \wedge I} \xi_{i, j+1}\right)\right)\right)
\end{aligned}
$$

and this proves the first claim of the proposition.

For $\nu_{i, j} \in \mathbb{R}$ with $i=t-J+1, \ldots, I$, and $j=t-i, \ldots, J-1$, we have

$$
\begin{aligned}
& \mathbb{E}\left[e^{\sum_{i=t-J+1}^{I} \sum_{j=t-i}^{J-1} \nu_{i, j} \xi_{i, j+1}} \mid \mathcal{T}_{t}\right] \\
= & \mathbb{E}\left[e^{\sum_{j=t-I}^{J-1} \sum_{i=t-j}^{I} \nu_{i, j} \xi_{i, j+1}} \mid \mathcal{T}_{t}\right] \\
= & \mathbb{E}\left[\mathbb{E}\left[e^{\sum_{j=t-I}^{J-1} \sum_{i=t-j}^{I} \nu_{i, j} \xi_{i, j+1}} \mid \boldsymbol{\Phi}, \mathcal{T}_{t}\right] \mid \mathcal{T}_{t}\right] \\
= & \mathbb{E}\left[\mathbb{E}\left[e^{\sum_{j=t-I}^{J-1} \sum_{i=t-j}^{I} \nu_{i, j} \xi_{i, j+1}} \mid \boldsymbol{\Phi}\right] \mid \mathcal{T}_{t}\right] \\
= & \mathbb{E}\left[e^{\sum_{j=t-I}^{J-1}\left(\left(\sum_{i=t-j}^{I} \nu_{i, j}\right) \Phi_{j}+0.5 \sum_{i=t-j}^{I}\left(\nu_{i, j}\right)^{2} \sigma_{j}^{2}\right)} \mid \mathcal{T}_{t}\right]
\end{aligned}
$$

and hence

$$
\begin{aligned}
& \log \mathbb{E}\left[e^{\sum_{i=t-J+1}^{I} \sum_{j=t-i}^{J-1} \nu_{i, j} \xi_{i, j+1}} \mid \mathcal{T}_{t}\right] \\
= & \left.\sum_{j=t-I}^{J-1} \sum_{i=t-j}^{I} \nu_{i, j} \phi_{j}^{(t)}+0.5 \sum_{j=t-I}^{J-1}\left(\sum_{i=t-j}^{I}\left(\nu_{i, j}\right)^{2}\left(\left(s_{j}^{(t)}\right)^{2}+\sigma_{j}^{2}\right)\right)+2 \sum_{t-j \leq i<k \leq I} \nu_{i, j} \nu_{k, j}\left(s_{j}^{(t)}\right)^{2}\right)
\end{aligned}
$$

which proves the second claim of the proposition.

\subsection{Proof of Proposition 2}

The proof goes by iteration. Since, for $t-j \leq I$,

$$
\frac{\left(s_{j}^{(t+1)}\right)^{2}}{\left(s_{j}^{(t)}\right)^{2}}+\frac{\left(s_{j}^{(t+1)}\right)^{2}}{\sigma_{j}^{2}}=1,
$$


we have

$$
\begin{aligned}
\phi_{j}^{(t+1)} & =\left(s_{j}^{(t+1)}\right)^{2}\left[\frac{\phi_{j}}{s_{j}^{2}}+\frac{1}{\sigma_{j}^{2}} \sum_{i=1}^{t-j-1} \xi_{i, j+1}+\frac{1}{\sigma_{j}^{2}} \xi_{t-j, j+1}\right] \\
& =\frac{\left(s_{j}^{(t+1)}\right)^{2}}{\left(s_{j}^{(t)}\right)^{2}} \phi_{j}^{(t)}+\frac{\left(s_{j}^{(t+1)}\right)^{2}}{\sigma_{j}^{2}} \xi_{t-j, j+1}=\beta_{j}^{(t+1)} \phi_{j}^{(t)}+\alpha_{j}^{(t+1)} \xi_{t-j, j+1}
\end{aligned}
$$

and (2.1) holds. By iterating for $l=2$, we obtain

$$
\phi_{j}^{(t+2)}=\beta_{j}^{(t+2)} \beta_{j}^{(t+1)} \phi_{j}^{(t)}+\beta_{j}^{(t+2)} \alpha_{j}^{(t+1)} \xi_{t-j, j+1}+\alpha_{j}^{(t+2)} \xi_{t+1-j, j+1}
$$

and then

$$
\phi_{j}^{(t+l)}=\phi_{j}^{(t)} \gamma_{j}^{(t+1: t+l)}+\sum_{q=1}^{l} \gamma_{j}^{(t+q+1: t+l)} \alpha_{j}^{(t+q)} \xi_{t-j+q-1, j+1} .
$$

Moreover, by using Proposition 1, a straightforward calculation gives $\mathbb{E}\left[\phi_{j}^{(t+l)} \mid \mathcal{T}_{t}\right]=\phi_{j}^{(t)}$ and

$$
\begin{aligned}
& \mathbb{E}\left[e^{\phi_{j}^{(t+l)}} \mid \mathcal{T}_{t}\right]
\end{aligned}
$$

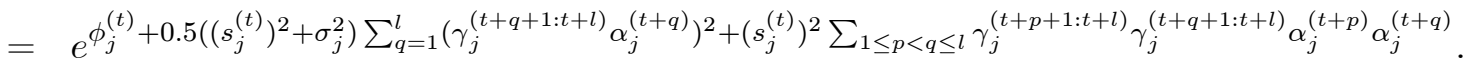

This completes the proof.

\subsection{Proof of Proposition 3}

For $i=t-J+1, \ldots, I$, we have

$$
R_{i, t}=C_{i, t-i}\left(e^{\sum_{j=t-i}^{J-1} \xi_{i, j+1}}-1\right)
$$

and

$$
R_{i, t}^{(d)}=\sum_{j=t-i}^{J-1} C_{i, j} \frac{e^{\xi_{i, j+1}}-1}{(1+r)^{j-t+i+1}}=C_{i, t-i} \sum_{j=t-i}^{J-1} e^{\sum_{l=t-i}^{j-1} \xi_{i, l+1}} \frac{e^{\xi_{i, j+1}}-1}{(1+r)^{j-t+i+1}} .
$$

By using Proposition 1, we obtain

$$
\mathbb{E}\left[R_{i, t} \mid \mathcal{T}_{t}\right]=C_{i, t-i}\left(\prod_{j=t-i}^{J-1} e^{\phi_{j}^{(t)}+0.5\left(\left(s_{j}^{(t)}\right)^{2}+\sigma_{j}^{2}\right)}-1\right)
$$

and

$$
\mathbb{E}\left[R_{i, t}^{(d)} \mid \mathcal{T}_{t}\right]=C_{i, t-i}\left(\sum_{j=t-i}^{J-1} \frac{1}{(1+r)^{j-t+i+1}}\left(e^{M_{t-i: j}^{(t)}+0.5 S_{t-i: j}^{(t)}}-e^{M_{t-i: j-1}^{(t)}+0.5 S_{t-i: j-1}^{(t)}}\right)\right)
$$




\subsection{Proof of Proposition 4}

The proof goes by backward induction. Let assume that, for $t=I, \ldots, i+J$,

$$
S C R_{i, t}=C_{i, t-i} e^{M_{t-i: J-1}^{(t)}} a_{i, t}
$$

where $a_{i, t}$ is a constant and $a_{i, i+J}=0$.

In view of (4.11), for $t=i+J-1$, the following equation holds

$$
\begin{aligned}
S C R_{i, i+J-1} & =\frac{1}{1+c}\left(q_{99.5 \%}\left(C_{i, J} \mid \mathcal{T}_{i+J-1}\right)-\mathbb{E}\left[C_{i, J} \mid \mathcal{T}_{i+J-1}\right]\right) \\
& =\frac{1}{1+c}\left(C_{i, J-1} e^{\phi_{J-1}^{(t+J-1)}+\varphi \sqrt{\left(\left(s_{J-1}^{(i+J-1)}\right)^{2}+\sigma_{J-1}^{2}\right)}}-C_{i, J-1} e^{\phi_{J-1}^{(t+J-1)}+0.5\left(\left(s_{J-1}^{(i+J-1)}\right)^{2}+\sigma_{J-1}^{2}\right)}\right) \\
& =\frac{C_{i, J-1} e^{\phi_{J-1}^{(t+J-1)}}}{1+c}\left(e^{\varphi \sqrt{\left(\left(s_{J-1}^{(i+J-1)}\right)^{2}+\sigma_{J-1}^{2}\right)}}-e^{0.5\left(\left(s_{J-1}^{(i+J-1)}\right)^{2}+\sigma_{J-1}^{2}\right)}\right)
\end{aligned}
$$

and hence

$$
a_{i, i+J-1}=\frac{1}{1+c}\left(e^{\varphi \sqrt{\left(\left(s_{J-1}^{(i+J-1)}\right)^{2}+\sigma_{J-1}^{2}\right)}}-e^{0.5\left(\left(s_{J-1}^{(i+J-1)}\right)^{2}+\sigma_{J-1}^{2}\right)}\right) .
$$

For $t<i+J-1$, we need for the calculation of $S C R_{i, t}$ to use the induction assumption. Let us evaluate successively each part of (4.11).

a) First we consider the conditional expected values of the ultimate claim $C_{i, J}$. From Proposition 1 , we easily obtain

$$
\mathbb{E}\left[C_{i, J} \mid \mathcal{T}_{t}\right]=C_{i, t-i} e^{M_{t-i: J-1}^{(t)}} e^{0.5 S_{t-i: J-1}^{(t)}}
$$

and

$$
\begin{aligned}
\mathbb{E}\left[C_{i, J} \mid \mathcal{T}_{t+1}\right] & =C_{i, t+1-i} e^{M_{t+1-i: J-1}^{(t+1)}} e^{0.5 S_{t+1-i: J-1}^{(t+1)}} \\
& =C_{i, t-i} e^{\xi_{i, t-i+1}+\sum_{j=t+1-i}^{J-1}\left(\beta_{j}^{(t+1)} \phi_{j}^{(t)}+\alpha_{j}^{(t+1)} \xi_{t-j, j+1}\right)} e^{0.5 S_{t+1-i: J-1}^{(t+1)}} \\
& =C_{i, t-i} e^{\sum_{j=t+1-i}^{J-1} \beta_{j}^{(t+1)} \phi_{j}^{(t)}} e^{\xi_{i, t-i+1}+\sum_{j=t+1-i}^{J-1} \alpha_{j}^{(t+1)}} \xi_{t-j, j+1} e^{0.5 S_{t+1-i: J-1}^{(t+1)}}
\end{aligned}
$$

b) Second we consider the conditional expected values of the future SCRs. Using the induction 
assumption, we have, for $l \geq t+1$,

$$
\begin{aligned}
& \mathbb{E}\left[S C R_{i, l} \mid \mathcal{T}_{t}\right] \\
= & a_{i, l} \mathbb{E}\left[C_{i, l-i} \prod_{j=l-i}^{J-1} e^{\phi_{j}^{(l)}} \mid \mathcal{T}_{t}\right] \\
= & a_{i, l} C_{i, t-i} \mathbb{E}\left[\prod_{j=t-i}^{l-i-1} e^{\xi_{i, j+1}} \prod_{j=l-i}^{J-1} e^{\left.\phi_{j}^{(t)} \gamma_{j}^{(t+1: l)}+\sum_{q=1}^{l-t} \gamma_{j}^{(t+q+1: l)} \alpha_{j}^{(t+q)} \xi_{t-j+q-1, j+1} \mid \mathcal{T}_{t}\right]}\right. \\
= & a_{i, l} C_{i, t-i} \prod_{j=l-i}^{J-1} e^{\phi_{j}^{(t)} \gamma_{j}^{(t+1: l)}} \mathbb{E}\left[\prod_{j=t-i}^{l-i-1} e^{\xi_{i, j+1}} \prod_{j=l-i}^{J-1} e^{\left.\sum_{q=1}^{l-t} \gamma_{j}^{(t+q+1: l)} \alpha_{j}^{(t+q)} \xi_{t-j+q-1, j+1} \mid \mathcal{T}_{t}\right]}\right. \\
= & a_{i, l} C_{i, t-i} \prod_{j=t-i}^{J-1} e^{\phi_{j}^{(t)}} \prod_{j=t-i}^{l-i-1} e^{0.5\left(\left(s_{j}^{(t)}\right)^{2}+\sigma_{j}^{2}\right)} \\
& \times \prod_{j-1}^{J-1} e^{0.5\left(\left(s_{j}^{(t)}\right)^{2}+\sigma_{j}^{2}\right) \sum_{q=1}^{l-t}\left(\gamma_{j}^{(t+q+1: l)} \alpha_{j}^{(t+q)}\right)^{2}+\left(s_{j}^{(t)}\right)^{2} \sum_{1 \leq p<q \leq l-t} \gamma_{j}^{(t+p+1: l)} \gamma_{j}^{(t+q+1: l)} \alpha_{j}^{(t+p)} \alpha_{j}^{(t+q)}} \\
& \quad C_{i, t-i} \prod_{j=t-i}^{J-1} e^{\phi_{j}^{(t)}\left[a_{i, l} \Sigma_{i, l, t}\right] .} \\
= &
\end{aligned}
$$

Hence

$$
c \sum_{l=t+1}^{i+J-1} \mathbb{E}\left[S C R_{i, l} \mid \mathcal{T}_{t}\right]=C_{i, t-i} \prod_{j=t-i}^{J-1} e^{\phi_{j}^{(t)}}\left[\sum_{l=t+1}^{i+J-1} c X_{i, l} \Sigma_{i, l, t}\right]
$$

and

$$
\begin{aligned}
c \sum_{l=t+1}^{i+J-1} \mathbb{E}\left[S C R_{i, l} \mid \mathcal{T}_{t+1}\right] & =C_{i, t+1-i} \prod_{j=t+1-i}^{J-1} e^{\phi_{j}^{(t+1)}}\left[\sum_{l=t+1}^{i+J-1} c a_{i, l} \Sigma_{i, l, t+1}\right] \\
& =C_{i, t-i} e^{\sum_{j=t+1-i}^{J-1} \beta_{j}^{(t+1)} \phi_{j}^{(t)}} e^{\xi_{i, t-i+1}+\sum_{j=t+1-i}^{J-1} \alpha_{j}^{(t+1)} \xi_{t-j, j+1}}\left[\sum_{l=t+1}^{i+J-1} c a_{i, l} \Sigma_{i, l, t+1}\right] .
\end{aligned}
$$

c) Third we aggregate the conditional expected values measurable with respect to $\mathcal{T}_{t}$

$$
c \sum_{l=t+1}^{i+J-1} \mathbb{E}\left[S C R_{i, l} \mid \mathcal{T}_{t}\right]+\mathbb{E}\left[C_{i, J} \mid \mathcal{T}_{t}\right]=C_{i, t-i} e^{\sum_{j=t-i}^{J-1} \phi_{j}^{(t)}} A_{i, t}^{(1)}
$$

with

$$
A_{i, t}^{(1)}=\sum_{l=t+1}^{i+J-1} c a_{i, l} \Sigma_{i, l, t}+e^{0.5 \sum_{j=t-i}^{J-1}\left(\left(s_{j}^{(t)}\right)^{2}+\sigma_{j}^{2}\right)}=\sum_{l=t+1}^{i+J-1} c a_{i, l} \Sigma_{i, l, t}+e^{0.5 S_{t-i: J-1}^{(t)}} .
$$

d) Fourth we aggregate the conditional expected values measurable with respect to $\mathcal{T}_{t+1}$ and derive the quantile of the sum given $\mathcal{T}_{t}$. Let

$c \sum_{l=t+1}^{i+J-1} \mathbb{E}\left[S C R_{i, l} \mid \mathcal{T}_{t+1}\right]+\mathbb{E}\left[C_{i, J} \mid \mathcal{T}_{t+1}\right]=C_{i, t-i} e^{\sum_{j=t+1-i}^{J-1} \beta_{j}^{(t+1)} \phi_{j}^{(t)}} e^{\xi_{i, t-i+1}+\sum_{j=t+1-i}^{J-1} \alpha_{j}^{(t+1)}} \xi_{t-j, j+1} A_{i, t}^{(2)}$ 
with

$$
A_{i, t}^{(2)}=\sum_{l=t+1}^{i+J-1} c a_{i, l} \Sigma_{i, l, t+1}+e^{0.5 \sum_{j=t+1-i}^{J-1}\left(\left(s_{j}^{(t+1)}\right)^{2}+\sigma_{j}^{2}\right)}=\sum_{l=t+1}^{i+J-1} c a_{i, l} \Sigma_{i, l, t+1}+e^{0.5 S_{t+1-i: J-1}^{(t+1)}}
$$

Moreover

$$
\begin{aligned}
& q_{99.5 \%}\left(e^{\xi_{i, t-i+1}+\sum_{j=t+1-i}^{J-1} \alpha_{j}^{(t+1)} \xi_{t-j, j+1}} \mid \mathcal{T}_{t}\right) \\
= & e^{\phi_{t-i}^{(t)}+\sum_{j=t+1-i}^{J-1} \alpha_{j}^{(t+1)} \phi_{j}^{(t)}} e^{\varphi \sqrt{\left(\left(s_{t-i}^{(t)}\right)^{2}+\sigma_{t-i}^{2}\right)+\sum_{j=t+1-i}^{J-1}\left(\alpha_{j}^{(t+1)}\right)^{2}\left(\left(s_{j}^{(t)}\right)^{2}+\sigma_{j}^{2}\right)}}
\end{aligned}
$$

and hence

$$
\begin{aligned}
& q_{99.5 \%}\left(c \sum_{l=t+1}^{i+J-1} \mathbb{E}\left[S C R_{i, l} \mid \mathcal{T}_{t+1}\right]+\mathbb{E}\left[C_{i, J} \mid \mathcal{T}_{t+1}\right] \mid \mathcal{T}_{t}\right) \\
= & C_{i, t-i} e^{\sum_{j=t-i}^{J-1} \phi_{j}^{(t)}} A_{i, t}^{(2)} e^{\varphi} \sqrt{\left(\left(\left(s_{t-i}^{(t)}\right)^{2}+\sigma_{t-i}^{2}\right)+\sum_{j=t+1-i}^{J-1}\left(\alpha_{j}^{(t+1)}\right)^{2}\left(\left(s_{j}^{(t)}\right)^{2}+\sigma_{j}^{2}\right)\right)} \\
= & C_{i, t-i} e^{\sum_{j=t-i}^{J-1} \phi_{j}^{(t)}} A_{i, t}^{(2)} \Xi_{i, t} .
\end{aligned}
$$

e) Fifth we gather the previous results and deduce that

$$
S C R_{i, t}=C_{i, t-i} \prod_{j=t-i}^{J-1} e^{\phi_{j}^{(t)}}\left[\frac{1}{1+c}\left(A_{i, t}^{(2)} \Xi_{i, t}-A_{i, t}^{(1)}\right)\right] .
$$

Therefore the constants $a_{i, t}$ satisfy the following recursive formulas

$$
a_{i, t}=\frac{1}{1+c}\left[c \sum_{l=t+1}^{i+J-1} a_{i, l}\left(\Sigma_{i, l, t+1} \Xi_{i, t}-\Sigma_{i, l, t}\right)+e^{0.5 S_{t+1-i: J-1}^{(t+1)}} \Xi_{i, t}-e^{0.5 S_{t-i: J-1}^{(t)}}\right] .
$$

Finally

$$
M V M_{i, t}=c \sum_{l=t}^{i+J-1} \mathbb{E}\left[S C R_{i, l} \mid \mathcal{T}_{t}\right]=c\left[\sum_{l=t}^{i+J-1} a_{i, l} \Sigma_{i, l, t}\right]\left[C_{i, t-i} \prod_{j=t-i}^{J-1} e^{\phi_{j}^{(t)}}\right] .
$$

This completes the proof.

\subsection{Proof of Proposition 5}

Let $n=I+J$ and, for $t=I, \ldots, n$,

$$
S_{t}=\sum_{i=1}^{I} C_{i,(t-i) \wedge J}=\sum_{i=1}^{t-J} C_{i, J}+\sum_{i=t-J+1}^{I} C_{i, t-i} .
$$

As for Proposition 4, the proof goes by backward induction. Let assume that, for $t=I, \ldots, I+J$,

$$
S C R_{t} \simeq \sum_{i=t-J+1}^{I} C_{i, t-i} e^{M_{t-i: J-1}^{(t)}} b_{i, t}
$$

where $b_{i, t}$ are constants which satisfy, for $j \geq i+J, b_{i, j}=0$. 
In view of (4.12), for $t=n-1=I+J-1$, the following equation holds

$$
\begin{aligned}
S C R_{n-1} & =\frac{1}{1+c}\left(q_{99.5 \%}\left(S_{n} \mid \mathcal{T}_{n-1}\right)-\mathbb{E}\left[S_{n} \mid \mathcal{T}_{n-1}\right]\right) \\
& =\frac{1}{1+c}\left(q_{99.5 \%}\left(C_{I, J} \mid \mathcal{T}_{n-1}\right)-\mathbb{E}\left[C_{I, J} \mid \mathcal{T}_{n-1}\right]\right) \\
& =\frac{1}{1+c}\left(C_{I, J-1} e^{\phi_{J-1}^{(n-1)}+\phi \sqrt{\left(\left(s_{J-1}^{(n-1)}\right)^{2}+\sigma_{J-1}^{2}\right)}}-C_{I, J-1} e^{\phi_{J-1}^{(n-1)}+0.5\left(\left(s_{J-1}^{(n-1)}\right)^{2}+\sigma_{J-1}^{2}\right)}\right) \\
& =\frac{C_{I, J-1} e^{\phi_{J-1}^{(n-1)}}}{1+c}\left(e^{\varphi \sqrt{\left(\left(s_{J-1}^{(n-1)}\right)^{2}+\sigma_{J-1}^{2}\right)}}-e^{0.5\left(\left(s_{J-1}^{(n-1)}\right)^{2}+\sigma_{J-1}^{2}\right)}\right)
\end{aligned}
$$

and

$$
b_{I, I+J-1}=\frac{1}{1+c}\left(e^{\varphi \sqrt{\left(\left(s_{J-1}^{(n-1)}\right)^{2}+\sigma_{J-1}^{2}\right)}}-e^{0.5\left(\left(s_{J-1}^{(n-1)}\right)^{2}+\sigma_{J-1}^{2}\right)}\right) .
$$

For $t<n-1$, we need for the calculation of $S C R_{t}$ to use the induction assumption. Let us evaluate successively each part of (4.12).

a) First we consider the conditional expected values of the ultimate claims $\sum_{i=1}^{I} C_{i, J}$. From Proposition 1, we easily obtain

$$
\begin{aligned}
\mathbb{E}_{[}\left[\sum_{i=1}^{I} C_{i, J} \mid \mathcal{T}_{t}\right] & =\mathbb{E}\left[S_{n} \mid \mathcal{T}_{t}\right]=\sum_{i=1}^{t-J} C_{i, J}+\sum_{i=t-J+1}^{I} \mathbb{E}\left[C_{i, J} \mid \mathcal{T}_{t}\right] \\
& =\sum_{i=1}^{t-J} C_{i, J}+\sum_{i=t-J+1}^{I} C_{i, t-i} e^{M_{t-i: J-1}^{(t)}+0.5 S_{t-i: J-1}^{(t)}}
\end{aligned}
$$

and

$$
\begin{aligned}
\mathbb{E}\left[S_{n} \mid \mathcal{T}_{t+1}\right]= & \sum_{i=1}^{t+1-J} C_{i, J}+\sum_{i=t+1-J+1}^{I} \mathbb{E}\left[C_{i, J} \mid \mathcal{T}_{t+1}\right] \\
= & \sum_{i=1}^{t-J} C_{i, J}+C_{t+1-J, J}+\sum_{i=t-J+2}^{I} C_{i, t+1-i} e^{M_{t+1-i: J-1}^{(t+1)}+0.5 S_{t+1-i: J-1}^{(t+1)}} \\
= & \sum_{i=1}^{t-J} C_{i, J}+C_{t+1-J, J-1} e^{\xi_{t+1-J, J}} \\
& +\sum_{i=t-J+2}^{I} C_{i, t-i} e^{\sum_{j=t+1-i}^{J-1} \beta_{j}^{(t+1)} \phi_{j}^{(t)}} e^{\xi_{i, t-i+1}+\sum_{j=t+1-i}^{J-1} \alpha_{j}^{(t+1)} \xi_{t-j, j+1}}\left[e^{\left.0.5 S_{t+1-i: J-1}^{(t+1)}\right] .}\right.
\end{aligned}
$$

b) Second we consider the conditional expected values of the future SCRs. Using the induction assumption, we have, for $l \geq t+1$,

$$
\mathbb{E}\left[S C R_{l} \mid \mathcal{T}_{t}\right]=\sum_{i=l-J+1}^{I} b_{i, l} \mathbb{E}\left[C_{i, l-i} e^{M_{l-i: J-1}^{(l)}} \mid \mathcal{T}_{t}\right]=\sum_{i=l-J+1}^{I} C_{i, t-i} e^{M_{t-i: J-1}^{(t)}\left[b_{i, l} \Sigma_{i, l, t}\right]}
$$

Hence

$$
\begin{aligned}
c \sum_{l=t+1}^{I+J-1} \mathbb{E}\left[S C R_{l} \mid \mathcal{T}_{t}\right] & =c \sum_{l=t+1}^{I+J-1} \sum_{i=l-J+1}^{I} C_{i, t-i} e^{M_{t-i: J-1}^{(t)}\left[b_{i, l} \Sigma_{i, l, t}\right]} \\
& =c \sum_{i=t-J+2}^{I} C_{i, t-i} e^{M_{t-i: J-1}^{(t)}} \sum_{l=t+1}^{i+J-1} b_{i, l} \Sigma_{i, l, t}
\end{aligned}
$$


and

$$
\begin{aligned}
& c \sum_{l=t+1}^{I+J-1} \mathbb{E}\left[S C R_{l} \mid \mathcal{T}_{t+1}\right]=c \sum_{l=t+1}^{I+J-1} \sum_{i=l-J+1}^{I} C_{i, t+1-i} e^{M_{t+1-i: J-1}^{(t+1)}\left[b_{i, l} \Sigma_{i, l, t+1}\right]} \\
& =c \sum_{i=t-J+2}^{I} C_{i, t+1-i} e^{M_{t+1-i: J-1}^{(t+1)}} \sum_{l=t+1}^{i+J-1} b_{i, l} \Sigma_{i, l, t+1} \\
& =\sum_{i=t-J+2}^{I} C_{i, t-i} e^{\sum_{j=t+1-i}^{J-1} \beta_{j}^{(t+1)} \phi_{j}^{(t)}} e^{\xi_{i, t-i+1}+\sum_{j=t+1-i}^{J-1} \alpha_{j}^{(t+1)}} \xi_{t-j, j+1}\left[\sum_{l=t+1}^{i+J-1} c b_{i, l} \Sigma_{i, l, t+1}\right]
\end{aligned}
$$

c) Third we aggregate the conditional expected values measurable with respect to $\mathcal{T}_{t}$

$$
\begin{aligned}
& c \sum_{l=t+1}^{I+J-1} \mathbb{E}\left[S C R_{l} \mid \mathcal{T}_{t}\right]+\mathbb{E}\left[S_{n} \mid \mathcal{T}_{t}\right] \\
= & c \sum_{i=t-J+2}^{I} C_{i, t-i} e^{M_{t-i: J-1}^{(t)}} \sum_{l=t+1}^{i+J-1} b_{i, l} \Sigma_{i, l, t}+\sum_{i=1}^{t-J} C_{i, J}+\sum_{i=t-J+1}^{I} C_{i, t-i} e^{M_{t-i: J-1}^{(t)}+0.5 S_{t-i: J-1}^{(t)}} \\
= & \sum_{i=1}^{t-J} C_{i, J}+\sum_{i=t-J+1}^{I} C_{i, t-i} e^{M_{t-i: J-1}^{(t)}} B_{i, t}^{(1)}
\end{aligned}
$$

with for $i=t-J+1, \ldots, I$

$$
B_{i, t}^{(1)}=\sum_{l=t+1}^{i+J-1} c b_{i, l} \Sigma_{i, l, t}+e^{0.5 S_{t-i: J-1}^{(t)}}
$$

d) Fourth we aggregate the conditional expected values measurable with respect to $\mathcal{T}_{t+1}$ and construct an approximation of the quantile of the sum given $\mathcal{T}_{t}$ by using the methodology presented in Section 3. We have

$$
\begin{aligned}
& c \sum_{l=t+1}^{I+J-1} \mathbb{E}\left[S C R_{l} \mid \mathcal{T}_{t+1}\right]+\mathbb{E}\left[S_{n} \mid \mathcal{T}_{t+1}\right] \\
= & \sum_{i=t-J+2}^{I} C_{i, t-i} e^{\sum_{j=t+1-i}^{J-1} \beta_{j}^{(t+1)} \phi_{j}^{(t)}} e^{\xi_{i, t-i+1}+\sum_{j=t+1-i}^{J-1} \alpha_{j}^{(t+1)} \xi_{t-j, j+1}}\left[\sum_{l=t+1}^{i+J-1} c b_{i, l} \Sigma_{i, l, t+1}\right]+\sum_{i=1}^{t-J} C_{i, J} \\
& +C_{t+1-J, J-1} e^{\xi_{t+1-J, J}} \\
& +\sum_{i=t-J+2}^{I} C_{i, t-i} e^{\sum_{j=t+1-i}^{J-1} \beta_{j}^{(t+1)} \phi_{j}^{(t)}} e^{\xi_{i, t-i+1}+\sum_{j=t+1-i}^{J-1} \alpha_{j}^{(t+1)} \xi_{t-j, j+1}}\left[e^{0.5 S_{t+1-i: J-1}^{(t+1)}}\right] \\
= & \sum_{i=1}^{t-J} C_{i, J}+\sum_{i=t-J+1}^{I} C_{i, t-i} e^{\sum_{j=t+1-i}^{J-1} \beta_{j}^{(t+1)} \phi_{j}^{(t)}} e^{\xi_{i, t-i+1}+\sum_{j=t+1-i}^{J-1} \alpha_{j}^{(t+1)}} \xi_{t-j, j+1} B_{i, t}^{(2)}
\end{aligned}
$$

with, for $i=t-J+2, \ldots, I$,

$$
B_{i, t}^{(2)}=\sum_{l=t+1}^{i+J-1} c b_{i, l} \Sigma_{i, l, t+1}+e^{0.5 S_{t+1-i: J-1}^{(t+1)}}
$$


and, for $i=t-J+1$,

$$
B_{t-J+1, t}^{(2)}=1 .
$$

We want to evaluate the quantiles of the following sum of log-normal random variables

$$
S^{(t)}=\sum_{i=t-J+1}^{I} \delta_{i}^{(t)} e^{Z_{i}^{(t)}}=\sum_{i=t-J+1}^{I}\left[C_{i, t-i} e^{\sum_{j=t+1-i}^{J-1} \beta_{j}^{(t+1)} \phi_{j}^{(t)}} B_{i, t}^{(2)}\right] e^{\xi_{i, t-i+1}+\sum_{j=t+1-i}^{J-1} \alpha_{j}^{(t+1)} \xi_{t-j, j+1}}
$$

with

$$
\delta_{i}^{(t)}=C_{i, t-i} e^{\sum_{j=t+1-i}^{J-1} \beta_{j}^{(t+1)} \phi_{j}^{(t)}} B_{i, t}^{(2)}, \quad Z_{i}^{(t)}=\xi_{i, t-i+1}+\sum_{j=t+1-i}^{J-1} \alpha_{j}^{(t+1)} \xi_{t-j, j+1} .
$$

Straightforward calculations give

$$
\begin{aligned}
\mathbb{E}\left[Z_{i}^{(t)} \mid \mathcal{T}_{t}\right] & =\phi_{t-i}^{(t)}+\sum_{j=t+1-i}^{J-1} \alpha_{j}^{(t+1)} \phi_{j}^{(t)} \\
\operatorname{Var}\left(Z_{i}^{(t)} \mid \mathcal{T}_{t}\right) & =\sigma_{Z_{i}^{(t)}}^{2}=\left(\left(s_{t-i}^{(t)}\right)^{2}+\sigma_{t-i}^{2}\right)+\sum_{j=t+1-i}^{J-1}\left(\alpha_{j}^{(t+1)}\right)^{2}\left(\left(s_{j}^{(t)}\right)^{2}+\sigma_{j}^{2}\right) \\
\operatorname{cov}\left(Z_{i}^{(t)}, Z_{l}^{(t)} \mid \mathcal{T}_{t}\right) & =\operatorname{Var}\left(Z_{i}^{(t)} \mid \mathcal{T}_{t}\right) \text { for } i \leq l .
\end{aligned}
$$

Let

$$
\Lambda^{(t)}=\sum_{i=t-J+1}^{I} \eta_{i}^{(t)} Z_{i}^{(t)}
$$

with

$$
\begin{aligned}
\eta_{i}^{(t)}= & \mathbb{E}\left[\delta_{i}^{(t)} \mid \mathcal{T}_{I}\right] e^{\mathbb{E}\left[Z_{i}^{(t)} \mid \mathcal{T}_{I}\right]+0.5 \operatorname{Var}\left(Z_{i}^{(t)} \mid \mathcal{T}_{I}\right)} \\
= & B_{i, t}^{(2)} \mathbb{E}\left[C_{i, t-i} e^{\sum_{j=t+1-i}^{J-1} \beta_{j}^{(t+1)} \phi_{j}^{(t)}} \mid \mathcal{T}_{I}\right] \\
& \times e^{\left(\phi_{t-i}^{(I)}+\sum_{j=t+1-i}^{J-1} \alpha_{j}^{(I+1)} \phi_{j}^{(I)}\right)+0.5\left(\left(\left(s_{t-i}^{(I)}\right)^{2}+\sigma_{t-i}^{2}\right)+\sum_{j=t+1-i}^{J-1}\left(\alpha_{j}^{(I+1)}\right)^{2}\left(\left(s_{j}^{(I)}\right)^{2}+\sigma_{j}^{2}\right)\right)}
\end{aligned}
$$

Note that $\eta_{i}^{(t)}$ is determined by using conditional expectations and conditional variance with respect to $\mathcal{T}_{I}$ and not $\mathcal{T}_{t}$ because this would have introduced additional terms linked to the $\phi_{t-i}^{(t)}$ and would have distorted the recursive form (7.20). From Proposition 1, we easily obtain

$$
\begin{aligned}
& \mathbb{E}\left[C_{i, t-i} e^{\sum_{j=t+1-i}^{J-1} \beta_{j}^{(t+1)} \phi_{j}^{(t)}} \mid \mathcal{T}_{I}\right] \\
= & C_{i, I-i} \mathbb{E}\left[e^{\sum_{j=I-i}^{t-i-1} \xi_{i, j+1}} e^{\sum_{j=t+1-i}^{J-1} \beta_{j}^{(t+1)}\left(\phi_{j}^{(I)} \gamma_{j}^{(I+1: t)}+\sum_{q=1}^{t-1} \gamma_{j}^{(I+q+1: t)} \alpha_{j}^{(I+q)} \xi_{I-j+q-1, j+1}\right)} \mid \mathcal{T}_{I}\right] \\
= & C_{i, I-i} e^{M_{I-i: t-i-1}^{(I)}+0.5 S_{I-i: t-i-1}^{(I)}} e^{\sum_{j=t+1-i}^{J-1} \beta_{j}^{(t+1)}\left(\phi_{j}^{(I)}+0.5 \beta_{j}^{(t+1)}\left(\left(s_{j}^{(I)}\right)^{2}+\sigma_{j}^{2}\right) \sum_{q=1}^{t-I}\left(\gamma_{j}^{(I+q+1: t)} \alpha_{j}^{(I+q)}\right)^{2}\right)} \\
& \times e^{\left.\sum_{j=t+1-i}^{J-1}\left(\beta_{j}^{(t+1)}\right)^{2}\left(s_{j}^{(I)}\right)^{2} \sum_{1 \leq p<q \leq t-I} \gamma_{j}^{(I+q+1: t)} \alpha_{j}^{(I+q)} \gamma_{j}^{(I+p+1: t)} \alpha_{j}^{(I+p)}\right)}
\end{aligned}
$$

and therefore

$$
\begin{aligned}
\eta_{i}^{(t)}= & B_{i, t}^{(2)} C_{i, I-i} e^{M_{I-i: t-i-1}^{(I)}+0.5 S_{I-i: t-i-1}^{(I)}} e^{\sum_{j=t+1-i}^{J-1} \beta_{j}^{(t+1)}\left(\phi_{j}^{(I)}+0.5\left(\left(s_{j}^{(I)}\right)^{2}+\sigma_{j}^{2}\right) \sum_{q=1}^{t-I}\left(\gamma_{j}^{(I+q+1: t)} \alpha_{j}^{(I+q)}\right)^{2}\right)} \\
& \times e^{\left.\sum_{j=t+1-i}^{J-1}\left(\beta_{j}^{(t+1)}\right)^{2}\left(s_{j}^{(I)}\right)^{2} \sum_{1 \leq p<q \leq t-I} \gamma_{j}^{(I+q+1: t)} \alpha_{j}^{(I+q)} \gamma_{j}^{(I+p+1: t)} \alpha_{j}^{(I+p)}\right)} \\
& \times e^{\left(\phi_{t-i}^{(I)}+\sum_{j=t+1-i}^{J-1} \alpha_{j}^{(t+1)} \phi_{j}^{(I)}\right)+0.5\left(\left(\left(s_{t-i}^{(I)}\right)^{2}+\sigma_{t-i}^{2}\right)+\sum_{j=t+1-i}^{J-1}\left(\alpha_{j}^{(t+1)}\right)^{2}\left(\left(s_{j}^{(I)}\right)^{2}+\sigma_{j}^{2}\right)\right)} .
\end{aligned}
$$


Then we derive

$$
\begin{aligned}
\mathbb{E}\left[\Lambda^{(t)} \mid \mathcal{T}_{t}\right] & =\sum_{i=t-J+1}^{I} \eta_{i}^{(t)} \mathbb{E}\left[Z_{i}^{(t)} \mid \mathcal{T}_{t}\right] \\
\mathbb{V} a r\left(\Lambda^{(t)} \mid \mathcal{T}_{t}\right) & =\sigma_{\Lambda^{(t)}}^{2}=\sum_{i=t-J+1}^{I}\left(\eta_{i}^{(t)}\right)^{2} \sigma_{Z_{i}^{(t)}}^{2}+2 \sum_{t-J+1 \leq i<l \leq I} \eta_{i}^{(t)} \eta_{l}^{(t)} \sigma_{Z_{i}^{(t)}}^{2}
\end{aligned}
$$

and

$$
\begin{aligned}
r_{i}^{(t)} & =\frac{\operatorname{cov}\left(Z_{i}^{(t)}, \Lambda^{(t)} \mid \mathcal{T}_{t}\right)}{\sigma_{Z_{i}^{(t)}} \sigma_{\Lambda^{(t)}}}=\sum_{l=t-J+1}^{I} \frac{\operatorname{cov}\left(Z_{i}^{(t)}, \eta_{l}^{(t)} Z_{l}^{(t)} \mid \mathcal{T}_{t}\right)}{\sigma_{Z_{i}^{(t)}} \sigma_{\Lambda^{(t)}}} \\
& =\frac{1}{\sigma_{Z_{i}^{(t)}} \sigma_{\Lambda^{(t)}}}\left(\sum_{l=t-J+1}^{i-1} \eta_{l}^{(t)} \sigma_{Z_{l}^{(t)}}^{2}+\sigma_{Z_{i}^{(t)}}^{2} \sum_{l=i}^{I} \eta_{l}^{(t)}\right) .
\end{aligned}
$$

The approximation $S^{l,(t)}$ of $S^{(t)}$ can be written

$$
S^{l,(t)}=\sum_{i=t-J+1}^{I} \delta_{i}^{(t)} e^{\mathbb{E}\left[Z_{i}^{(t)} \mid \mathcal{T}_{t}\right]+0.5\left(1-\left(r_{i}^{(t)}\right)^{2}\right) \sigma_{Z_{i}^{(t)}}^{2}+r_{i}^{(t)} \sigma_{Z_{i}^{(t)}} \Phi^{-1}(U)}
$$

where $U$ is uniform distributed random variable and the approximation of the quantile is then given by

$$
\begin{aligned}
q_{99.5 \%}\left(S^{l,(t)} \mid \mathcal{T}_{t}\right) & =\sum_{i=t-J+1}^{I} \delta_{i}^{(t)} e^{\mathbb{E}\left[Z_{i}^{(t)} \mid \mathcal{I}_{t}\right]+0.5\left(1-\left(r_{i}^{(t)}\right)^{2}\right) \sigma_{Z_{i}^{2}}^{2}+r_{i}^{(t)} \sigma_{Z_{i}^{(t)} \varphi}} \\
& =\sum_{i=t-J+1}^{I} C_{i, t-i} e^{M_{t-i: J-1}^{(t)} B_{i, t}^{(2)} \Xi_{i, t}^{S}}
\end{aligned}
$$

where

$$
\Xi_{i, t}^{S}=e^{0.5\left(1-\left(r_{i}^{(t)}\right)^{2}\right) \sigma^{2}} Z_{i}^{(t)}+r_{i}^{(t)} \sigma_{Z_{i}^{(t)} \varphi} .
$$

e) Fifth we gather the previous results and deduce that

$$
S C R_{t} \simeq \sum_{i=t-J+1}^{I} C_{i, t-i} e^{M_{t-i: J-1}^{(t)}}\left[\frac{1}{1+c}\left(B_{i, t}^{(2)} \Xi_{i, t}^{S}-B_{i, t}^{(1)}\right)\right]=\sum_{i=t-J+1}^{I} C_{i, t-i} e^{M_{t-i: J-1}^{(t)}} b_{i, t}
$$

where the constants $b_{i, t}$ satisfy the following recursive formulas

$$
\begin{aligned}
b_{i, t} & =\frac{1}{1+c}\left[\left(\sum_{l=t+1}^{i+J-1} c b_{i, l} \Sigma_{i, l, t+1}+e^{0.5 S_{t+1-i: J-1}^{(t+1)}}\right) \Xi_{i, t}^{S}-\sum_{l=t+1}^{i+J-1} c b_{i, l} \Sigma_{i, l, t}-e^{0.5 S_{t-i: J-1}^{(t)}}\right] \\
& =\frac{1}{1+c}\left[c \sum_{l=t+1}^{i+J-1} b_{i, l}\left(\Sigma_{i, l, t+1} \Xi_{i, t}^{S}-\Sigma_{i, l, t}\right)+e^{\left.0.5 S_{t+1-i: J-1}^{(t+1)} \Xi_{i, t}^{S}-e^{0.5 S_{t-i: J-1}^{(t)}}\right] .}\right.
\end{aligned}
$$


Finally we obtain

$$
\begin{aligned}
M V M_{t} & =c \sum_{j=t}^{I+J-1} \mathbb{E}\left[S C R_{j} \mid \mathcal{T}_{t}\right]=c \sum_{l=t}^{I+J-1} \mathbb{E}\left[\sum_{i=l-J+1}^{I} C_{i, l-i} e^{M_{l-i: J-1}^{(l)}} b_{i, l} \mid \mathcal{T}_{t}\right] \\
& =c \sum_{l=t}^{I+J-1} \sum_{i=l-J+1}^{I} b_{i, l} \mathbb{E}\left[\sum_{i=l-J+1}^{I} C_{i, l-i} e^{M_{l-i: J-1}^{(l)} \mid \mathcal{T}_{t}}\right] \\
& =c \sum_{l=t}^{I+J-1} \sum_{i=l-J+1}^{I} b_{i, l} \Sigma_{i, l, t}\left[C_{i, t-i} \prod_{j=t-i}^{J-1} e^{\phi_{j}^{(t)}}\right] .
\end{aligned}
$$

This completes the proof.

\subsection{Proofs of Proposition 6 and 7}

The proofs go by backward induction and follow the same lines as that given in the previous proof. In order to shorten the paper, the proofs are not given here but they are, however, available upon request.

\section{References}

[1] Bonnard, R., Margetts, S., 2008. Practical Market Value Margins, GIRO.

[2] Bühlmann, H., De Felice, M. Gisler, A., Moriconi, F., 2009. Recursive credibility formula for chain ladder factors and the claims development result. Astin Bulletin 39, 275-306.

[3] Bühlmann, H. and Gisler, A., 2005. A Course in Credibility Theory and its Applications. Springer.

[4] Daya, M., 2011. Market Value Margins for a non-life insurance company under Solvency II. Available under http://www.ressourcesactuarielles.net/C12574E200674F5B/0/3180D668739449BDC1257887001FF301

[5] Dhaene, J., Denuit, M., Goovaerts, M., Kaas, R., Vyncke, D., 2002a. The concept of comonotonicity in actuarial science and finance: theory. Insurance: Mathematics and Economics $31,3-33$.

[6] Dhaene, J., Denuit, M., Goovaerts, M., Kaas, R., Vyncke, D., 2002b. The concept of comonotonicity in actuarial science and finance: applications. Insurance: Mathematics and Economics 31, 133-161.

[7] Haslip, G., 2010. Solvency II: Taking a risk. The actuary, December 2010.

[8] Hertig, J., 1985. A statistical approach to the IBNR-reserves in marine insurance. Astin Bulletin 15, 171-183.

[9] International Actuarial Association IAA, 2009. Measurement of liabilities for insurance contracts: current estimates and risk margins. Available under www.actuaries.org.

[10] Kaas, R., Dhaene, J., Goovaerts, M., 2000. Upper and lower bounds for sums of random variables. Insurance: Mathematics and Economics 27, 151-168. 
[11] Merz, M., Wuthrich, M.V., 2007. Prediction error of the expected claims development result. Swiss Assoc. Act. , 1, 117-137.

[12] Merz, M., Wuthrich, M.V., 2010. Paid-incurred chain claims reserving method. Insurance: Mathematics and Economics 46, 568-579.

[13] Ohlsson E., Lauzeningks J., 2009. The one-year non-life insurance risk. Insurance: Mathematics and Economics 45, 203-208.

[14] Salzmann R., Wuthrich, M.V., 2010. Cost-of-capital margin for a general insurance liability runoff. Astin Bulletin 40, 415-451.

[15] Vanduffel, S., Hoedemakers, T., Dhaene, J., 2005. Comparing approximations for risk measures of sums of non-independent lognormal random variables. North American Actuarial Journal $9,71-82$.

[16] Vanduffel, S., Chen, X., Dhaene, J., Goovaerts, M., Henrard, L. Kaas, R., 2008. Optimal approximations for risk measures of sums of lognormals based on conditional expectations. Journal of Computational and Applied Mathematics 221, 202-218

[17] Wuthrich, M.V., 2007. Using a Bayesian approach for claims reserving. Variance 1, 292-301.

[18] Wuthrich, M.V., Embrechts, P., Tsanakas, A., 2011. Risk margin for a non-life insurance run-off. Preprint available under http://www.math.ethz.ch/ wueth/. 


\begin{tabular}{|c|c|c|c|c|c|c|c|c|c|c|c|c|c|c|c|c|c|}
\hline \multirow{2}{*}{$\begin{array}{c}\text { a.y. } \\
i\end{array}$} & \multicolumn{17}{|c|}{ development year $j$} \\
\hline & 0 & 1 & 2 & 3 & 4 & 5 & 6 & 7 & 8 & 9 & 10 & 11 & 12 & 13 & 14 & 15 & 16 \\
\hline 1 & 13109 & 20355 & 21337 & 22043 & 22401 & 22658 & 22997 & 23158 & 23492 & 23664 & 23699 & 23904 & 23960 & 23992 & 23994 & 24001 & 24002 \\
\hline 2 & 14457 & 22038 & 22627 & 23114 & 23238 & 23312 & 23440 & 23490 & 23964 & 23976 & 24048 & 24111 & 24252 & 24538 & 24540 & 24550 & \\
\hline 3 & 16075 & 22672 & 23753 & 24052 & 24206 & 24757 & 24786 & 24807 & 24823 & 24888 & 24986 & 25401 & 25681 & 25705 & 25732 & & \\
\hline 4 & 15682 & 23464 & 24465 & 25052 & 25529 & 25708 & 25752 & 25770 & 25835 & 26075 & 26082 & 26146 & 26150 & 26167 & & & \\
\hline 5 & 16551 & 23706 & 24627 & 25573 & 26046 & 26115 & 26283 & 26481 & 26701 & 26718 & 26724 & 26728 & 26735 & & & & \\
\hline 6 & 15439 & 23796 & 24866 & 25317 & 26139 & 26154 & 26175 & 26205 & 26764 & 26818 & 26836 & 26959 & & & & & \\
\hline 7 & 14629 & 21645 & 22826 & 23599 & 24992 & 25434 & 25476 & 25549 & 25604 & 25709 & 25723 & & & & & & \\
\hline 8 & 17585 & 26288 & 27623 & 27939 & 28335 & 28638 & 28715 & 28759 & 29525 & 30302 & & & & & & & \\
\hline 9 & 17419 & 25941 & 27066 & 27761 & 28043 & 28477 & 28721 & 28878 & 28948 & & & & & & & & \\
\hline 10 & 16665 & 25370 & 26909 & 27611 & 27729 & 27861 & 29830 & 29844 & & & & & & & & & \\
\hline 11 & 15471 & 23745 & 25117 & 26378 & 26971 & 27396 & 27480 & & & & & & & & & & \\
\hline 12 & 15103 & 23393 & 26809 & 27691 & 28061 & 29183 & & & & & & & & & & & \\
\hline 13 & 14540 & 22642 & 23571 & 24127 & 24210 & & & & & & & & & & & & \\
\hline 15 & 13967 & 21515 & 22603 & & & & & & & & & & & & & & \\
\hline 16 & 12930 & 20111 & & & & & & & & & & & & & & & \\
\hline 17 & 12539 & & & & & & & & & & & & & & & & \\
\hline
\end{tabular}

Table 3: Cumulative payments $C_{i, j}=\sum_{l=0}^{j} X_{i, l}, i+j \leq 17$ 